EPJ manuscript No.

(will be inserted by the editor)

FZJ-IKP-TH-2009-4,JLAB-THY-09-935

\title{
Neutral pion photoproduction at high energies
}

\author{
A. Sibirtsev ${ }^{1,2}$, J. Haidenbauer ${ }^{3,4}$, S. Krewald ${ }^{3,4}$, U.-G. Meißner ${ }^{1,3,4}$ and A.W. Thomas ${ }^{5,6}$ \\ 1 Helmholtz-Institut für Strahlen- und Kernphysik (Theorie) und Bethe Center for Theoretical Physics, Universität Bonn, D-53115 Bonn, \\ Germany \\ 2 Excited Baryon Analysis Center (EBAC), Thomas Jefferson National Accelerator Facility, Newport News, Virginia 23606, USA \\ 3 Institut für Kernphysik and Jülich Center for Hadron Physics, Forschungszentrum Jülich, D-52425 Jülich, Germany \\ 4 Institute of Advanced Simulations, Forschungszentrum Jülich, D-52425 Jülich, Germany \\ 5 Theory Center, Thomas Jefferson National Accelerator Facility, 12000 Jefferson Ave., Newport News, Virginia 23606, USA \\ ${ }^{6}$ College of William and Mary, Williamsburg, VA 23187, USA
}

Received: date / Revised version: date

\begin{abstract}
A Regge model with absorptive corrections is employed in a global analysis of the world data on the reactions $\gamma p \rightarrow \pi^{0} p$ and $\gamma n \rightarrow \pi^{0} n$ for photon energies from 3 to $18 \mathrm{GeV}$. In this region resonance contributions are expected to be negligible so that the available experimental information on differential cross sections and single- and double polarization observables at $-t \leq 2 \mathrm{GeV}^{2}$ allows us to determine the non-resonant part of the reaction amplitude reliably. The model amplitude is then used to predict observables for photon energies below $3 \mathrm{GeV}$. A detailed comparison with recent data from the CLAS and CB-ELSA Collaborations in that energy region is presented. Furthermore, the prospects for determining the $\pi^{0}$ radiative decay width via the Primakoff effect from the reaction $\gamma p \rightarrow \pi^{0} p$ are explored.
\end{abstract}

PACS. 11.55.Jy Regge formalism - 13.60.Le Meson production - 13.60.-r Photon and charged-lepton interactions with hadrons - 25.20.Lj Photoproduction reactions

\section{Introduction}

Recently we have completed [1] a systematic analysis of positive and negative pion photoproduction at invariant collision energies $\sqrt{s}>2 \mathrm{GeV}$. The main purpose of our study was to inspect whether the presently available data indicate any evidence for excitations of baryons with masses up to $3 \mathrm{GeV}$ or higher and to examine the issue of which observables might be most suitable for further pertinent investigations. The analyis was based on a Regge model with absorptive corrections that took into account the $\rho, b_{1}$, and $a_{2}$ trajectories and pion exchange. Free parameters of the amplitudes were fixed in a global fit of the world data on differential cross section and single polarization observables available at high energies, i.e. at $\sqrt{s}>3 \mathrm{GeV}$. In this region resonance contributions are expected to be negligible so that the available experimental information allows one to determine the non-resonant part of the reaction amplitude reliably. The model amplitude was then used to predict observables at energies below $3 \mathrm{GeV}$. Differences between the model predictions and data in this energy region were systematically examined as possible signals for the presence of excited baryons.

In the present paper we extend this analysis to neutral pion photoproduction. Guided by our previous study of charged pion photoproduction [1] we use again a gauge invariant Regge model, which now comprises Regge pole and cut amplitudes for $\rho, \omega$, and $b_{1}$ exchanges. Again, the free parameters of the model are fixed in a global fit to high energy data. Specifically, in our fit we include data on $\gamma p \rightarrow \pi^{0} p$ differential cross sections and single and double polarization observables available in the energy range $3 \leq E_{\gamma} \leq 18 \mathrm{GeV}$ but with the restriction $-t \leq 2 \mathrm{GeV}^{2}$. Those data were obtained around or before 1980. After that we proceed to analyse data on neutral pion photoproduction on the proton collected recently by the CLAS Collaboration at JLab [2] and by the CB-ELSA Collaboration in Bonn [3,4] in the energy region $2 \leq E_{\gamma} \leq 3 \mathrm{GeV}$.

A strong motivation for studying neutral pion photoproduction is provided by the occurence of the well-known Primakoff effect [5]. This effect is connected with the contribution of the one-photon exchange amplitude and manifests itself through a large differential cross section at very forward angles. The onephoton exchange amplitude, in turn, is directly connected with the $\pi^{0}$ radiative decay width and proportional to the charge of the target $Z$. The $\pi^{0} \rightarrow \gamma \gamma$ decay amplitude is related to symmetry breaking through the axial anomaly and reveals one of the fundamental properties of QCD [6,7,8,9,10,11,12, 13].

The fact that the differential cross section due to the Primakoff amplitude is proportional to $Z^{2}$ initiated strong activities in the determination of the $\pi^{0}$-meson radiative decay from measurements with nuclear targets [14, 15, 16, 17, 18, 19]. In, deed, most of the results [15, 16,17,18] on the neutral pion lifetime, given by the PDG [20], were obtained by utilizing 
A. Sibirtsev et. al: Neutral pion photoproduction at high energies

the Primakoff effect. A very precise experiment (PrimEx) on the determination of the $\pi^{0} \rightarrow \gamma \gamma$ decay width from $\pi^{0}$-meson photoproduction on nuclear targets is presently performed at JLab [21].

It was argued [14,21,22], however, that pion absorption on nuclei as well as the interference between the one-photon exchange and the nuclear amplitude may complicate the data analysis and significantly affect the accuracy of the results obtained on the $\pi^{0}$-meson lifetime. While the one photon exchange amplitude is explicitly given by theory, the evaluation of the nuclear amplitudes requires a precise knowledge of the elementary amplitude on a nucleon and the spectral function of the target nucleus. Although the $Z^{2}$-argument clearly favors nuclear targets, this advantage might be completely counterbalanced by the benefit of the $\pi^{0}$-meson photoproduction on the proton where one has a much better handle on the hadronic part of the production amplitude. Therefore, in the present paper we will re-examine [23] the prospects for determining the $\pi^{0}$ radiative decay width from neutral pion photoproduction on a proton target.

The paper is organized as follows. In Sect. 2 we formulate the reaction amplitudes. The parameters of the global fit to high energy data are given in Sect. 3. The analysis of neutral pion photoproduction at high and low energies is given in Sects. 4 and 5, respectively. Results for the ratio of the $\gamma n \rightarrow \pi^{0} n$ and $\gamma p \rightarrow \pi^{0} p$ differential cross section and the total cross section for the neutral pion photoproduction from the proton are presented in Sect. 6. In this section we also examine the energy dependence of the data for fixed four-momentum transfer. In Sect. 7 we discuss the Primakoff effect on the proton target and explore the prospects for future experiments. The paper ends with a short Summary.

\section{Reaction amplitudes}

Guided by our previous analysis of charged pion photoproduction [1] we use a gauge invariant Regge model which combines the Regge pole and cut amplitudes for $\rho, \omega$ and $b_{1}$ exchanges. At high energies the interactions before and after the basic Regge pole exchange mechanisms are essentially elastic or diffractive scattering described by Pomeron exchange. Such a scenario can be related to the distorted wave approximation and provides a well defined formulation [24, 25, 26, 27, 28,29] for constructing Regge cut amplitudes. This approach, which can also be derived in an eikonal formalism [30] with $s$-channel unitarity [31], is followed in our work. Detailed discussions about the non-diffractive multiple scattering corrections involving intermediate states which differ from the initial and final states and the relevant Reggeon unitarity equations are given in Refs. [24,32,33, 34]. For simplicity we do not consider these much more involved mechanisms which would increase significantly the number of parameters to be fitted.

We use the $t$-channel parity conserving helicity amplitudes $F_{i}(i=1, \ldots, 4)$. Here $F_{1}$ and $F_{2}$ are the natural and unnatural spin-parity $t$-channel amplitudes to all orders in $s$, respectively. $F_{3}$ and $F_{4}$ are the natural and unnatural $t$-channel amplitudes to leading order in $s$.
Each Regge pole helicity amplitude is parameterized as

$$
F(s, t)=\pi \beta(t) \frac{1+\mathcal{S} \exp [-i \pi \alpha(t)]}{\sin [\pi \alpha(t)] \Gamma[\alpha(t)]}\left[\frac{s}{s_{0}}\right]^{\alpha(t)-1},
$$

where $s$ is the invariant collision energy squared, $t$ is the squared four-momentum transfer and $s_{0}=1 \mathrm{GeV}^{2}$ is a scaling parameter that allows us to define a dimensionless amplitude. Furthermore, $\beta(t)$ is a residue function, $\mathcal{S}$ is the signature factor and $\alpha(t)$ is the Regge trajectory.

From Eq. (1), we see that the factor $\sin [\pi \alpha(t)]$ would generate poles at $t \leq 0$ when $\alpha(t)$ assumes the values $0,-1, \ldots$. The function $\Gamma[\alpha(t)]$ is introduced to suppress those poles that lie in the scattering region because

$$
\Gamma[\alpha(t)] \Gamma[1-\alpha(t)]=\frac{\pi}{\sin [\pi \alpha(t)]} .
$$

The structure of the vertex function $\beta(t)$ of Eq. (1) is defined by the quantum numbers of the particles at the interaction vertex, similar to the usual particle exchange Feyman diagram.

Both natural and unnatural parity particles can be exchanged in the $t$-channel. The naturalness $\mathcal{N}$ for natural $(\mathcal{N}=+1)$ and unnatural $(\mathcal{N}=-1)$ parity exchanges is defined as

$$
\begin{gathered}
\mathcal{N}=+1 \text { if } P=(-1)^{J}, \\
\mathcal{N}=-1 \text { if } P=(-1)^{J+1},
\end{gathered}
$$

where $P$ and $J$ are the parity and spin of the particle, respectively. Furthermore, in Regge theory each exchange is denoted by a signature factor $\mathcal{S}= \pm 1$ defined as $[24,35,36]$

$$
\mathcal{S}=P \times \mathcal{N}=(-1)^{J}
$$

which enters Eq. (1).

To proceed further we should specify the trajectories that contribute to neutral pion photoproduction. Note that the mechanisms for charged and neutral pion photoproduction are different. Indeed, for charged pion photoproduction pion exchange dominates at small $-t$ while $\omega$-exchange is forbidden altogether. For $\pi^{0}$-meson photoproduction just the opposite is the case.

One of the significant differences between the data on neutral and charged pion photoproduction is the presence of the dip in the $\gamma p \rightarrow \pi^{0} p$ differential cross sections at the squared four-momentum transfer $t \simeq-0.5 \mathrm{GeV}^{2}$. A similar dip is also observed in other reactions. For instance, in the $\pi^{-} p \rightarrow \pi^{0} n$ reaction such a dip results from the $\rho$-exchange amplitude and its position is related to the $\rho$-trajectory [37].

Therefore, one expects that $\rho$-exchange might dominate the $\gamma p \rightarrow \pi^{0} p$ reaction too. Indeed, the square of the amplitude of Eq. (1) for the $\rho$-exchange is proportional to

$$
|F(s, t)|^{2} \propto 1-\cos [\pi \alpha(t)],
$$

which has a zero at $t \simeq-0.6 \mathrm{GeV}^{2}$, when taking the $\rho$-trajectory as obtained recently in a fit [37] to the available data for the $\pi^{-} p \rightarrow \pi^{0} n$ reaction. Note that the additional contributions to the reaction amplitude $\left(\omega, b_{1}\right)$ can move this zero closer to experimentally observed value.

In the $\gamma p \rightarrow \pi^{0} p$ reaction there is no difference between the $\rho$ and $\omega$-exchanges if their trajectories are the same. Thus in 
Table 1. Correspondence between $t$-channel pole exchanges and the helicity amplitudes $F_{i}(i=1 \div 3)$. Here $P$ is parity, $J$ the spin, $I$ the isospin, $G$ the $G$-parity, $\mathcal{N}$ the naturalness and $\mathcal{S}$ the signature factor.

\begin{tabular}{|c|c|c|c|c|c|c|c|}
\hline$F_{i}$ & $P$ & $J$ & $I$ & $G$ & $\mathcal{N}$ & $\mathcal{S}$ & Exchange \\
\hline$F_{1}$ & -1 & 1 & 1 & +1 & +1 & -1 & $\rho$ \\
$F_{1}$ & -1 & 1 & 0 & +1 & +1 & -1 & $\omega$ \\
$F_{2}$ & +1 & 1 & 1 & +1 & -1 & -1 & $b_{1}$ \\
$F_{3}$ & -1 & 1 & 1 & +1 & +1 & -1 & $\rho$ \\
$F_{3}$ & -1 & 1 & 0 & +1 & +1 & -1 & $\omega$ \\
\hline
\end{tabular}

some previous studies [38] both contributions were considered as just one exchange amplitude. However, in our study we treat $\rho$ and $\omega$ exchanges separately, because any possible difference in the amplitudes might play a role in describing observables [39. 40,41. For instance it could allow us to fix the ratio of the $\gamma n \rightarrow \pi^{0} n$ and $\gamma p \rightarrow \pi^{0} p$ differential cross sections. Note that in these two reactions the contributions from the isovector exchange enter with a different sign, whereas the isoscalar exchange is the same in both cases.

The contributions of the $\rho$ and $\omega$-exchanges to the reaction amplitudes $F_{i}$ are indicated in Table 1 together with the relevant quantum numbers. Both $\rho$ and $\omega$ have natural parity and contribute to $F_{1}$ and $F_{3}$. It was argued [40] that if there are no other contributions one would expect that the photon asymmetry $\Sigma$ would be predominantly +1 . (However, note that $\Sigma$ vanishes at forward and backward directions [42].) This can be easily understood when considering the relations between the observables and the $t$-channel parity conserving helicity amplitudes given in the Appendix A. Experimental data [43, 44] available at high energies shows that the asymmetry indeed is consistent with +1 for $t$ values above -0.4 and below -1.1 . For $-1<t<-0.4 \mathrm{GeV}^{2}$ there is a dip in the asymmetry and at $t \simeq-0.5 \mathrm{GeV}^{2}$ it drops to $\Sigma \simeq 0.7$. Thus, one needs to take into account additional trajectories that yield contributions to the unnatural-parity amplitudes $F_{2}$ and $F_{4}$. Indeed, the leading $b_{1}$ trajectory, which we already used in the analysis of charged pion photoproduction contributes to $F_{2}$, cf. Table 1 and, therefore, we include it here too.

As we discussed in Ref. [1] the empirical information about those trajectories that yield contributions to $F_{4}$ is very sparse. Hence, we neglected this amplitude in our analysis of charged pion photoproduction. For the same reason we also neglect $F_{4}$ in the present study. Our decision can be justified by considering the data $45,46,47$ on the target $(T)$ and recoil $(P)$ asymmetry, available for the $\gamma p \rightarrow \pi^{0} p$ reaction at photon energies above $4 \mathrm{GeV}$. Since they fulfill roughly $T \simeq P$ one can conclude that $F_{4} \simeq 0$, based on the relations given in Eqs. (17) and (18) of Appendix A. We will discuss this point below.

The trajectories are taken in the following linear form,

$$
\alpha(t)=\alpha_{0}+\alpha^{\prime} t
$$

where the intercept and slope for the $\rho$ and $b_{1}$ trajectories are taken over from analyses of other reactions [1, 24, 37, 48]. Explicitly we have for the $\rho$ and $b_{1}$ trajectories

$$
\alpha_{\rho}=0.53+0.8 t
$$

Table 2. Parameterization of the residue functions $\beta(t)$ for the amplitudes $F_{i}, \quad(i=1,2,3)$. Here $c_{i j}$ is the coupling constant where the double index refers to the amplitude $i$ and the type of exchange $j$, as specified in the Table.

\begin{tabular}{|l|l|c|c|}
\hline & $\beta(t)$ & Exchange & $j$ \\
\hline \multicolumn{3}{|c|}{ Pole amplitudes } \\
\hline$F_{1}$ & $c_{11}$ & $\rho$ & 1 \\
$F_{1}$ & $c_{12}$ & $\omega$ & 2 \\
\hline$F_{2}$ & $c_{23} t$ & $b_{1}$ & 3 \\
\hline$F_{3}$ & $c_{31} t$ & $\rho$ & 1 \\
$F_{3}$ & $c_{32} t$ & $\omega$ & 2 \\
\hline \multicolumn{3}{|c|}{$\operatorname{Cut}$ amplitudes } \\
\hline$F_{1}$ & $c_{14} \exp \left[d_{4} t\right]$ & $\rho$ & 4 \\
$F_{1}$ & $c_{15} \exp \left[d_{5} t\right]$ & $\omega$ & 5 \\
$F_{1}$ & $c_{16} \exp \left[d_{6} t\right]$ & $b_{1}$ & 6 \\
\hline$F_{2}$ & $c_{24} t \exp \left[d_{4} t\right]$ & $\rho$ & 4 \\
$F_{2}$ & $c_{25} t \exp \left[d_{5} t\right]$ & $\omega$ & 5 \\
$F_{2}$ & $c_{26} t \exp \left[d_{6} t\right]$ & $b_{1}$ & 6 \\
\hline$F_{3}$ & $c_{34} t \exp \left[d_{4} t\right]$ & $\rho$ & 4 \\
$F_{3}$ & $c_{35} t \exp \left[d_{5} t\right]$ & $\omega$ & 5 \\
$F_{3}$ & $c_{36} t \exp \left[d_{6} t\right]$ & $b_{1}$ & 6 \\
\hline
\end{tabular}

$$
\alpha_{b_{1}}=0.51+0.8 t
$$

The $\omega$-trajectory was parameterized by Eq. (6) with the slope $\alpha^{\prime}=0.8 \mathrm{GeV}^{-2}, i$. $e$. the same value as for other trajectories. The parameter $\alpha_{0}$ for $\omega$-exchange was fixed by a fit to the data.

The residue functions $\beta(t)$ used in our analysis are compiled in Table 2. They are similar to the ones used in some of the previous analyses [39,49]. These residues are slightly different from those applied in our study of charged pion photoproduction [1] because here we use explicitely the $\Gamma$ function in the amplitude parameterization of Eq. (1).

In defining the Regge cut amplitudes we use the following parameterization based on the absorption model [35, 39,50,51, 52]

$$
F(s, t)=\frac{\pi \beta(t)}{\log \left(s / s_{0}\right)} \frac{1+\mathcal{S} \exp \left[-i \pi \alpha_{c}(t)\right]}{\sin \left[\pi \alpha_{c}(t)\right] \Gamma\left[\alpha_{c}(t)\right]}\left[\frac{s}{s_{0}}\right]^{\alpha_{c}(t)-1},
$$

with the trajectories defined by

$$
\alpha_{c}=\alpha_{0}+\frac{\alpha^{\prime} \alpha_{P}^{\prime} t}{\alpha^{\prime}+\alpha_{P}^{\prime}},
$$

where $\alpha_{0}$ and $\alpha^{\prime}$ are taken from the pole trajectory given by Eqs. (6) and (7), and $\alpha_{P}^{\prime}=0.2 \mathrm{GeV}^{-2}$ is the slope of the Pomeron trajectory. The residue functions $\beta(t)$ of Eq. (8) are given in Table 2

In the very forward direction of the $\gamma p \rightarrow \pi^{0} p$ reaction there is an interference of the $F_{1}$ amplitude with the one-photon exchange amplitude, which is known as Primakoff effect [5]. This effect allows to determine the radiative decay width of the $\pi^{0}$ meson. However, the experimental resolution of the $\gamma p \rightarrow \pi^{0} p$ 
Table 3. Parameters of the model. Here $c_{i j}$ is the coupling constant for the $i$-th amplitude and the type $j$ of exchange, $d_{j}$ is a cut-off parameter for the Regge cut amplitude.

\begin{tabular}{|c|c|c|c|c|}
\hline$j$ & \multicolumn{3}{|c|}{$c_{i j}$} & $d_{j}$ \\
& $i=1$ & $i=2$ & $i=3$ & \\
\hline 1 & -12.0 & - & -15.9 & - \\
2 & 41.4 & - & -56.1 & - \\
3 & - & 8.1 & - & - \\
4 & -2572 & 1174 & -6278 & 3.59 \\
5 & -18.9 & -4.8 & 37.5 & 0.64 \\
6 & 3055 & -1403 & 7526 & 3.65 \\
\hline
\end{tabular}

data available presently is insufficient to resolve the one-photon exchange amplitude. Thus, we omitted the interference region, i. e. $|t|<0.04 \mathrm{GeV}^{2}$ from the fit in order to fix the $F_{1}$ amplitude. But we add the one-photon exchange amplitude lateron and compare the results with the $\gamma p \rightarrow \pi^{0} p$ differential cross sections available at very forward direction.

The relations between the observables analyzed in our study and the $t$-channel helicity amplitudes are summarized in Appendix A. The relation between the $F_{i}$, the $s$-channel helicity amplitudes and the invariant amplitudes are given in Appendix B.

\section{Parameters of the model}

The resulting parameters of the model are listed in Table 3 . The achieved $\chi^{2} / \operatorname{dof}$ amounts to 1.4. We find that there are some inconsistencies between data from different experiments. Thus, it is not possible to improve the confidence level of our global analysis unless these inconsistent data are removed from the data base. However, it is difficult to specify sensible criteria for pruning the data base.

The intercept of the $\omega$-trajectory at $t=0$ obtained from the fit is $\alpha_{\omega}=0.641 \pm 0.003$, which indeed differs from that obtained for the $\rho$-trajectory. The coupling constants listed in Table 3 show that in case of the cut amplitudes there is some compensation between the $\rho, \omega$ and $b_{1}$-exchange contributions. However, tiny differences in the trajectories are reflected in very different couplings for the cut amplitudes. Furthermore, we find that the solution is very sensitive to the differential cross section in the vicinity of the dip. Indeed the dip structure results from the pole amplitudes. Since there are many data available around the dip, i.e. at $t \simeq-0.5 \mathrm{GeV}^{2}$, the parameters are well constrained by these data and the solution turns out to be stable.

In order to avoid any dependence of the fit on the starting values of the parameters we have used the random walk method to construct the initial parameter vector and we have repeated the minimization procedure. This allows us to practically exclude that we obtain just a local minimum. Furthermore, an additional examination is has been done by exploring the results for the parameters correlation matrix in order to find out how unique the found minimum is.
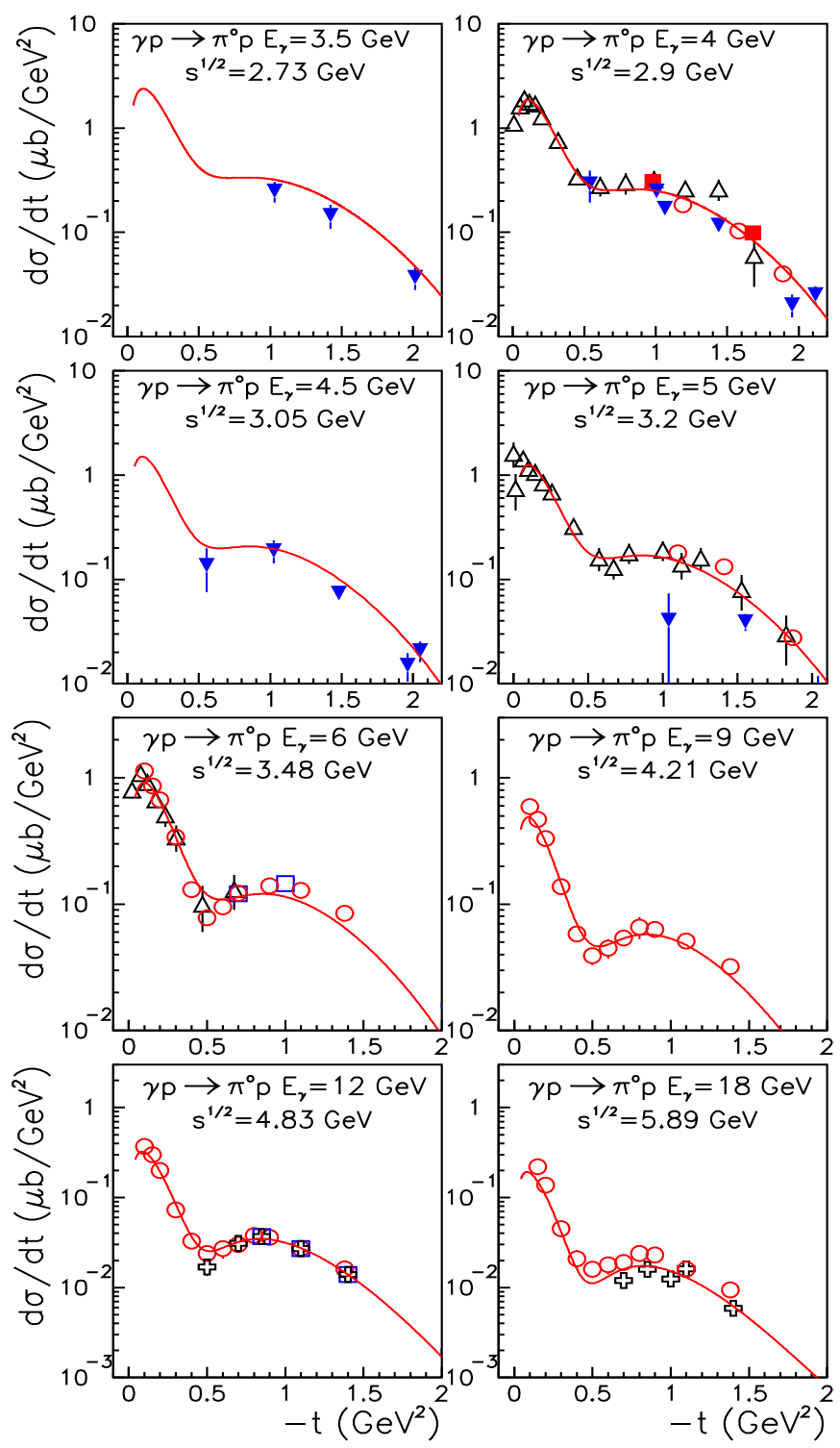

Fig. 1. Differential cross section for $\gamma p \rightarrow \pi^{0} p$ as a function of $-t$ at different photon energies $E_{\gamma}$ or invariant collision energies $\sqrt{s}$. The data are taken from Refs. [53] (filled inverse triangles), [54] (open triangles), [55 56] (filled squares), [57,44] (open circles), [58] (open squares) and [59] (crosses). The solid lines show the results of our model calculation.

\section{Results of the fit}

Our results for the $\gamma p \rightarrow \pi^{0} p$ differential cross sections at photon energies above $3 \mathrm{GeV}$ are presented in Fig. 1 . The model reproduces the data quite well. As we discussed previously the data indeed suggest a minimum or shoulder around the value $t=-0.5 \mathrm{GeV}^{2}$, which was not observed in the differential cross sections for the $\gamma p \rightarrow \pi^{+} n$ and $\gamma n \rightarrow \pi^{-} p$ reactions. Furthermore, the dip becomes more pronounced with increasing photon energy.

In Fig. 22 we display the data on the polarized photon asymmetry available for the reaction $\gamma p \rightarrow \pi^{0} p$ at photon energies above $3 \mathrm{GeV}$. The data [57,44] at the energies $E_{\gamma}=4,6$, and 

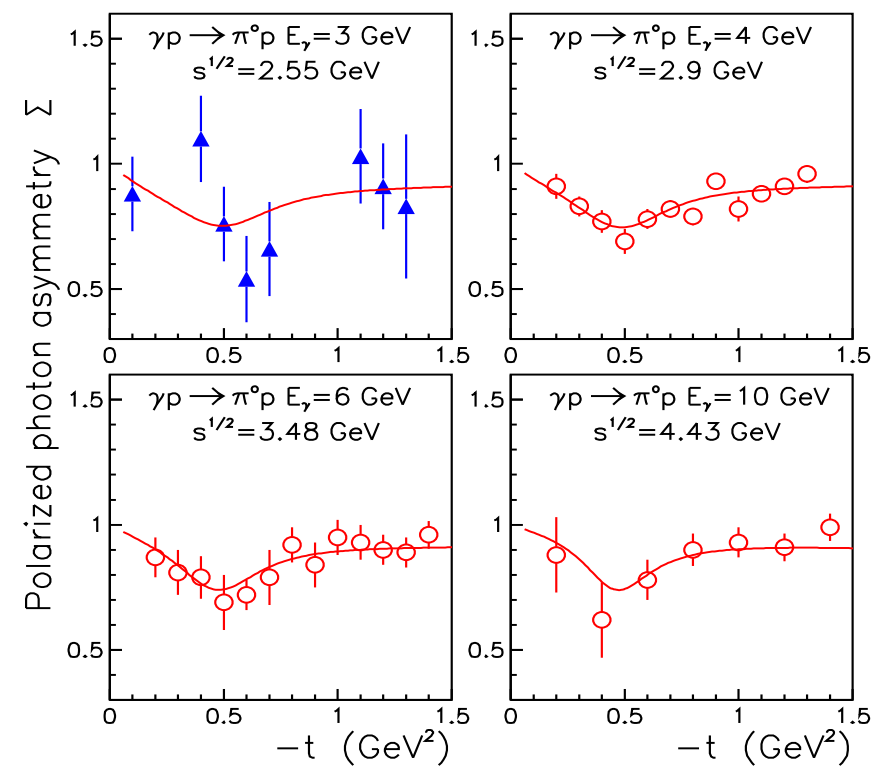

Fig. 2. Polarized photon asymmetry for $\gamma p \rightarrow \pi^{0} p$ as a function of $-t$ at different photon energies $E_{\gamma}$ or invariant collision energies $\sqrt{s}$. The data are taken from Refs. [43] (filled triangles) and [57 44] (open circles). The solid lines show the results of our model calculation.

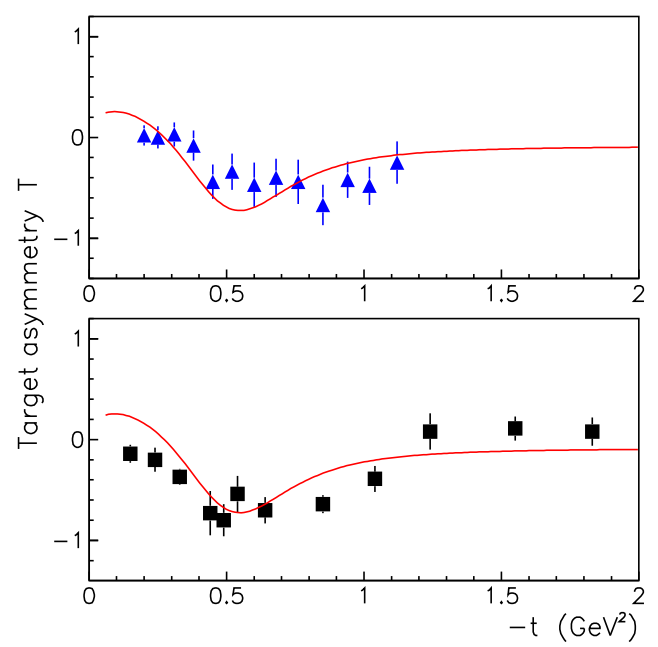

Fig. 3. Target asymmetry for $\gamma p \rightarrow \pi^{0} p$ as a function of $-t$ at photon energy $E_{\gamma}=4 \mathrm{GeV}$ or invariant collision energy $\sqrt{s}=2.9 \mathrm{GeV}$. The triangles are data from Ref. [46], while the squares are from Ref. [45]. The solid lines show the results of our model calculation.

$10 \mathrm{GeV}$ are sufficiently precise and clearly indicate a dip around $t \simeq-0.5 \mathrm{GeV}^{2}$. The Regge calculations reproduce the experimental results resonably well.

Next we take a look at the data available for the target $(T)$ and recoil $(P)$ asymmetries. Fig. 3 shows experimental results on the target asymmetry of the $\gamma p \rightarrow \pi^{0} p$ reaction at a photon energy of $4 \mathrm{GeV}$. The data [45,46] at the same energy are from independent measurements. The solid lines are the result of the Regge calculations. They are in agreement with the data within experimental uncertainties.

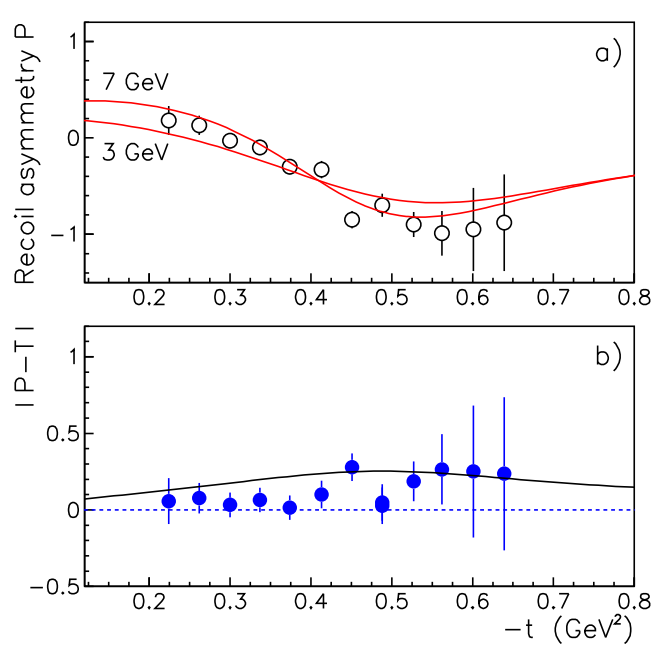

Fig. 4. a) Recoil asymmetry for $\gamma p \rightarrow \pi^{0} p$ as a function of $-t$. Open circles are the data [47] obtained at photon energies from 3 to $7 \mathrm{GeV}$. The lines show the Regge calculations at photon energies 3 and $7 \mathrm{GeV}$. b) Illustration of the Worden inequality given by Eq. (10). Closed circles are the difference between $P$ taken from experiment [47] and $P$ given by the Regge calculation. The solid line is $1-\Sigma$ with the polarized photon asymmetry taken from the calculations. The dashed line indicates the case $|P-T|=0$.

The open circles in Fig. 4la) are experimental results for the recoil asymmetry in the reaction $\gamma p \rightarrow \pi^{0} p$ for incident photon energies between 3 and $7 \mathrm{GeV}$ [47]. These data allow us to examine whether the target and recoil asymmetries are different. This issue was considered in Ref. [47] via a direct comparison of experimental results [45,46, 47] available for the target and recoil asymmetries. It was argued that $T \neq P$, so that there must be higher order contributions to the $\gamma p \rightarrow \pi^{0} p$ reaction. Indeed following Eqs. (17) and (18) the amplitude $F_{4}$ is not negligible in such a case.

However, as remarked in Ref. [47], the measurements of the target and recoil asymmetries were done at different energies and $P$ is averaged over photon energies ranging from 3 to $7 \mathrm{GeV}$. In particular, it was emphasized that without real calculations any conclusions remain quite speculative. Now we can investigate this issue in more detail. The two lines in Fig. (4h) show the Regge results for $E_{\gamma}=3 \mathrm{GeV}$ and $E_{\gamma}=7 \mathrm{GeV}$. Indeed the asymmetries $P$ obtained at the two energies are slightly different. The calculations reproduce the data fairly well and from that we conclude that there are no solid arguments to claim that $T \neq P$ at high energies and thus to speculate about any significance of the $F_{4}$ amplitude.

A further examination of $F_{4}$ can by done by applying the Worden inequality [29] given by

$$
|P-T| \leq 1-\Sigma
$$

and shown in the Fig. 4b). Here the closed circles indicate the difference between the experimental results [47] for $P$ and the calculation for $T$. The solid line is the difference $1-\Sigma$ with the polarized photon asymmetry taken from the calculation. Note that in this case the Regge results are in good agreement with the data on the $T$ and $\Sigma$ asymmetries. Fig. (4)illustrates that the 
inequality [29] given by Eq. (10) is satisfied within the experimental uncertainties.

\section{Predictions at energies below $3 \mathrm{GeV}$}

In this section we compare our predictions with older data for energies below $3 \mathrm{GeV}$ but also with the most recent experimental results [2,3] for differential cross sections collected by the CLAS Collaboration at JLab and by CB-ELSA in Bonn. Both latter experiments cover the energy range up to $E_{\gamma} \simeq 3 \mathrm{GeV}$. As pointed out in Ref. [2] the JLab results disagree with the CB-ELSA measurements at forward angles. It will be interesting to inspect the observed discrepancy with regard to the predictions by the Regge model. One knows from the case of charged pion photoproduction say, that the Regge phenomenology works well for forward angles even down to $E_{\gamma} \simeq 2 \mathrm{GeV}$.

As stressed in many studies [24,52,29], the Regge theory is phenomenological in nature. There is no solid theoretical derivation that allows us to establish explicitly the ranges of $t$ and $s$ where this formalism is applicable. Since there are several well-known nucleon resonances [20] in the energy range up to $\sqrt{s} \simeq 2.6 \mathrm{GeV}$, identified in partial wave analyses [60,61, 62,63,64] of pion-nucleon scattering, we expect that deviations of our predictions from the data will start to show up for energies from $E_{\gamma} \simeq 3 \mathrm{GeV}$ downwards. But it will be interesting to see whether and in which observables such discrepancies indeed occur.

We also present results utilizing the amplitudes from the partial wave analysis (PWA) of the GWU Group [65, 66, 67, [68], which was recently extended up to $\sqrt{s} \simeq 2.55 \mathrm{GeV}$ [2]. The results we display are based on the current solution taken from the interactive program SAID [69]. The interesting question here is whether there is an energy region where the PWA results are in agreement with the Regge calculations. This would be an indication that the PWA solution might have approached the high energy limit given by Regge phenomenology, at least in terms of the considered observables.

In Fig. 5 we display differential cross sections for $\gamma p \rightarrow \pi^{0} p$ at photon energies from 2.02 to $3 \mathrm{GeV}$. This energy region corresponds to invariant collision energies of $2.16 \leq \sqrt{s} \leq 2.55 \mathrm{GeV}$. As expected with decreasing energy the predictions of the Regge model differ more and more from the data. But at least down to roughly $\sqrt{s}=2.34 \mathrm{GeV}$ the results are still fairly well in line with the experimental information, considering the variations between the existing measurements. Note that the Regge model reproduces the strong rise of the cross section for very small angles, as seen in data from Ref. [54] at $\sqrt{s}=2.55 \mathrm{GeV}$, very accurately.

It is evident from Fig. 5 that there is a systematic disagreement between the CLAS results [2] (filled triangles) and the ELSA measurement (filled circles) at the higher energies, especially in the forward direction. With regard to our Regge results there is no clear preference for any of the two measurements. However, at least in the vicinity of the dip or shoulder our results are definitely more in line with the ELSA measurement. We would like to emphasize that neither the CLAS nor the ELSA data were included in our fit.

One can see from Fig. [5] that the GWU PWA [65,66,67, 68], shown by the dashed lines, reproduces the data from JLAB (to
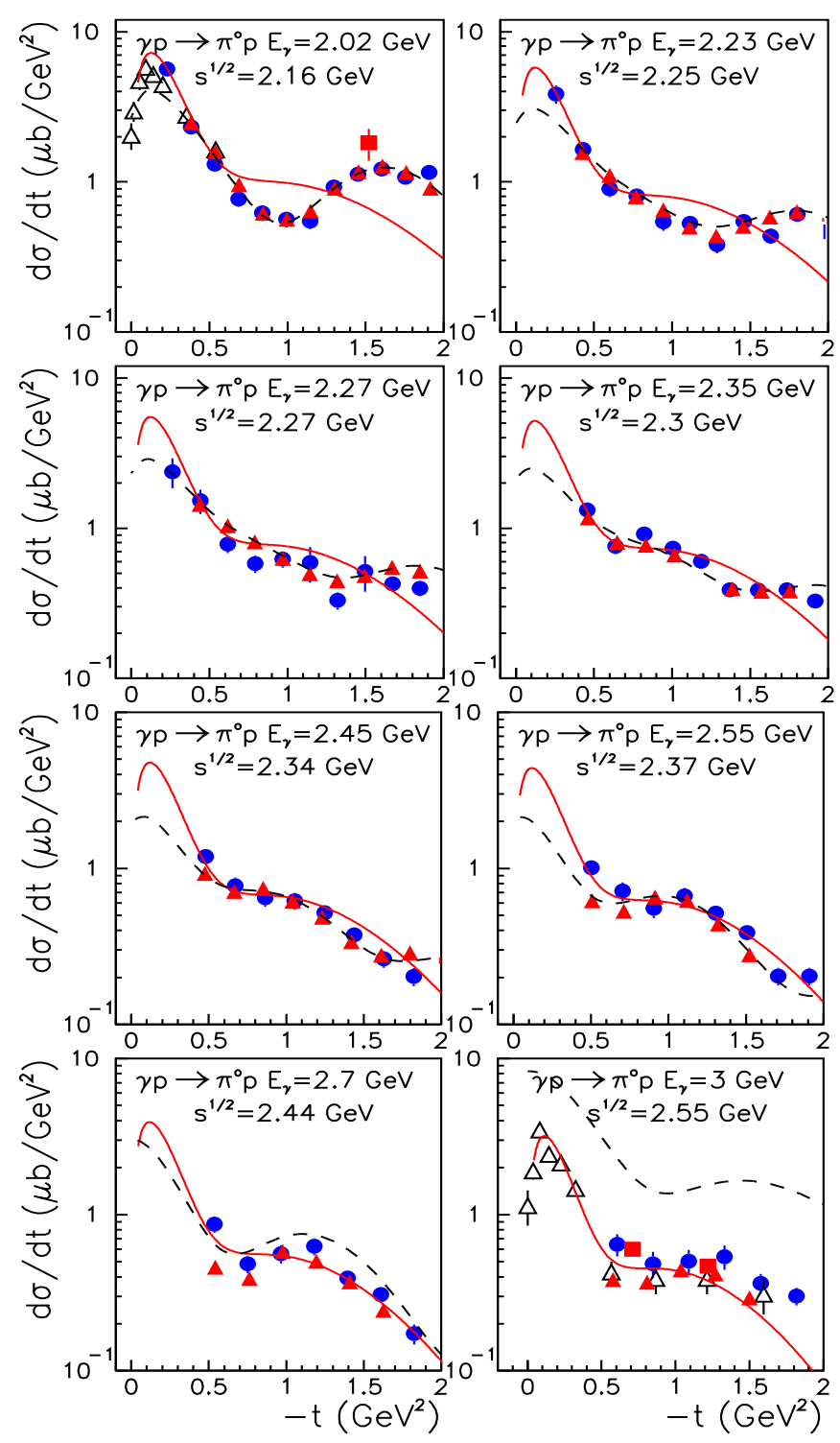

Fig. 5. Differential cross section for $\gamma p \rightarrow \pi^{0} p$ as a function of $-t$ at different photon energies $E_{\gamma}$ or invariant collision energies $\sqrt{s}$. The data are taken from Refs. [2] (filled triangles), [3] (filled circles), [54] (open triangles) and [55:56] (filled squares). The solid lines show the results of our model calculation. The dashed lines are the results based on the GWU PWA [69].

which it was fitted) very well up to photon energies of 2.55 $\mathrm{GeV}$ or invariant energies of $\sqrt{s}=2.37 \mathrm{GeV}$. Also, for invariant collisions energies around $2.34 \simeq 2.37 \mathrm{GeV}$ the GWU PWA results and the Regge calculations are similar, at least qualitatively and for the range $0.5 \leq-t \leq 1.2 \mathrm{GeV}^{2}$. It is important to note that our model does not include any resonance contribution. The GWU PWA indicates the presence of the $G_{39}(2400)$ $\Delta$-resonance at the upper end of the fit to $\pi N$ elastic scattering data [68]. But it remains unclear whether this resonance also has a noticable impact on their results for neutral pion photoproduction.

The polarized photon asymmetry, measured [70] at photon energies from 2.1 to $2.75 \mathrm{GeV}$, is shown in Fig. 6. Unfortu- 

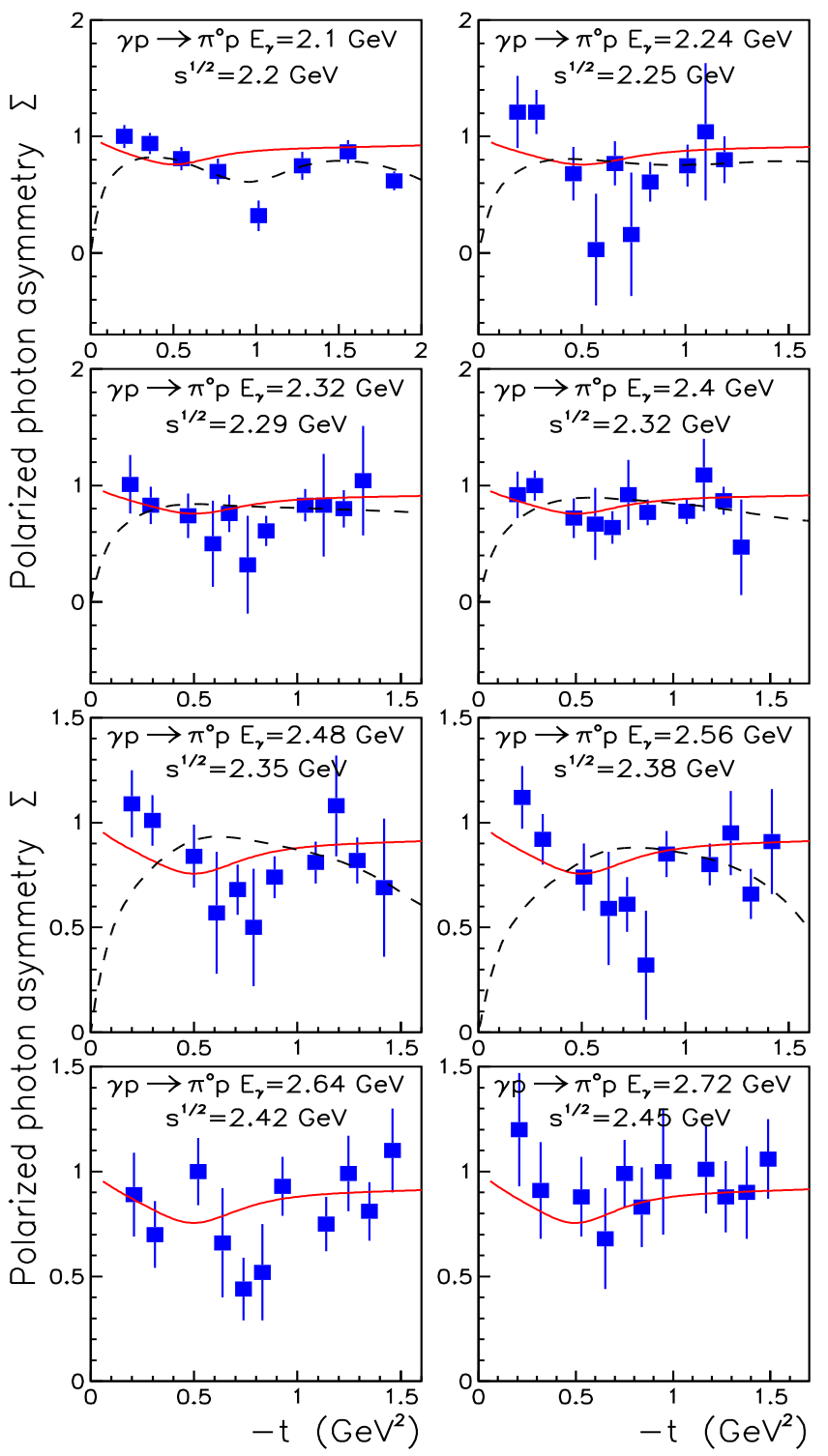

Fig. 6. Polarized photon asymmetry for $\gamma p \rightarrow \pi^{0} p$ as a function of $-t$ at different photon energies $E_{\gamma}$ or invariant collision energies $\sqrt{s}$. The data are taken from Refs. [70]. The solid lines show the results of our model calculation. The dashed lines are the results based on the GWU PWA [69].

nately, the experimental results are afflicted by large uncertainties and, therefore, do not allow us to draw any more quantitative conclusions on the reliability of the Regge predictions. But the results are roughly in line with the data over the whole energy region. The GWU PWA is in reasonable agreement with the data at $2.1 \mathrm{GeV}$, but develops a qualitatively different behavior with increasing energy. The data seem to indicate the presence of a dip at around $t \simeq-0.8 \mathrm{GeV}^{2}$ at almost all shown energies. The Regge model produces such a dip, but near the value $t \simeq-0.5 \mathrm{GeV}^{2}$, while the results from GWU PWA exhibit a dip structure only at the lowest energy considered.

Fig. 7 shows target and recoil asymmetries in the $\gamma p \rightarrow \pi^{0} p$ reaction, measured [70] at photon energies of 2 and $2.1 \mathrm{GeV}$. The data on the target asymmetry have quite small uncertainties
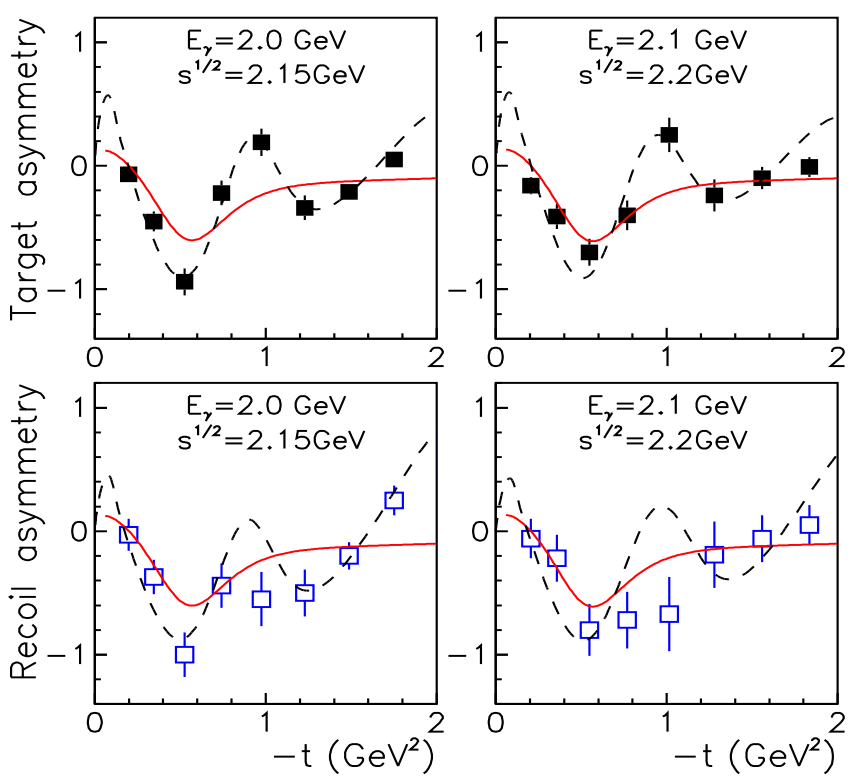

Fig. 7. Target (filled squares) and recoil (open squares) asymmetries for $\gamma p \rightarrow \pi^{0} p$ as a function of $-t$ at different photon energies $E_{\gamma}$ or invariant collision energies $\sqrt{s}$. The data are taken from Refs. [70]. The solid lines show the results of our model calculation. The dashed lines are the results based on the GWU PWA [69].
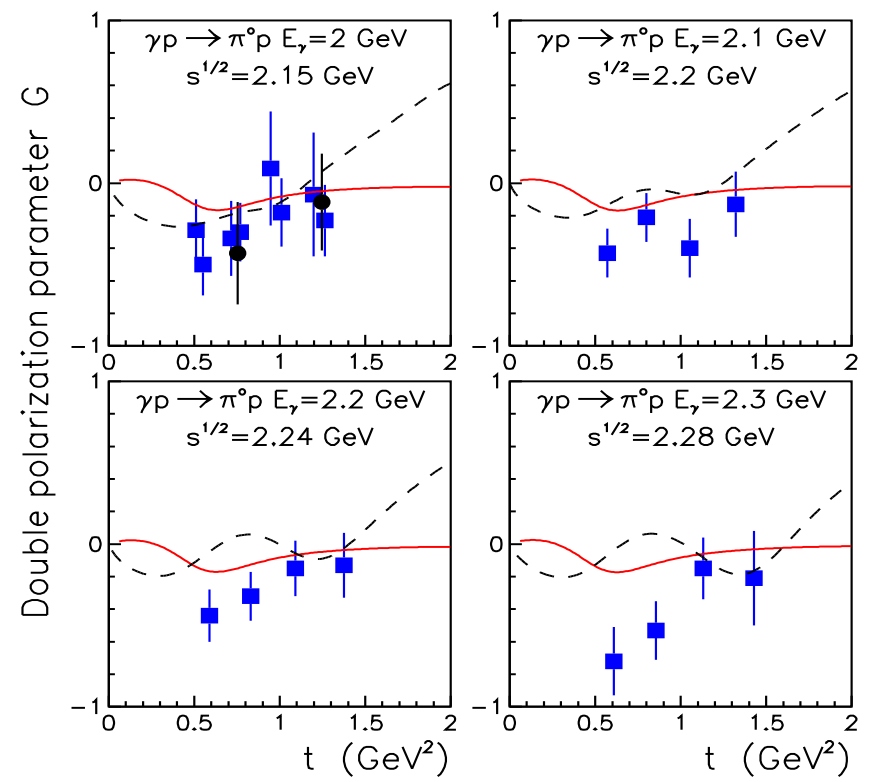

Fig. 8. Double polarization parameter $G$ for $\gamma p \rightarrow \pi^{0} p$ as a function of $-t$ given at different photon energies $E_{\gamma}$. The circles are results from Ref. [45], while the squares show the data from Ref. [74]. The solid lines show the results of our model calculation. The dashed lines are the results based on the GWU PWA [69].

and exhibit a significant variation with four-momentum transfer squared. Apparently, at these energies $T \neq P$. The Regge calculations reproduce the recoil and target asymmetry roughly, but only at very forward angles. On the other hand, the GWU PWA describes $T$ as well as $P$ fairly well over the considered $t$ range. 

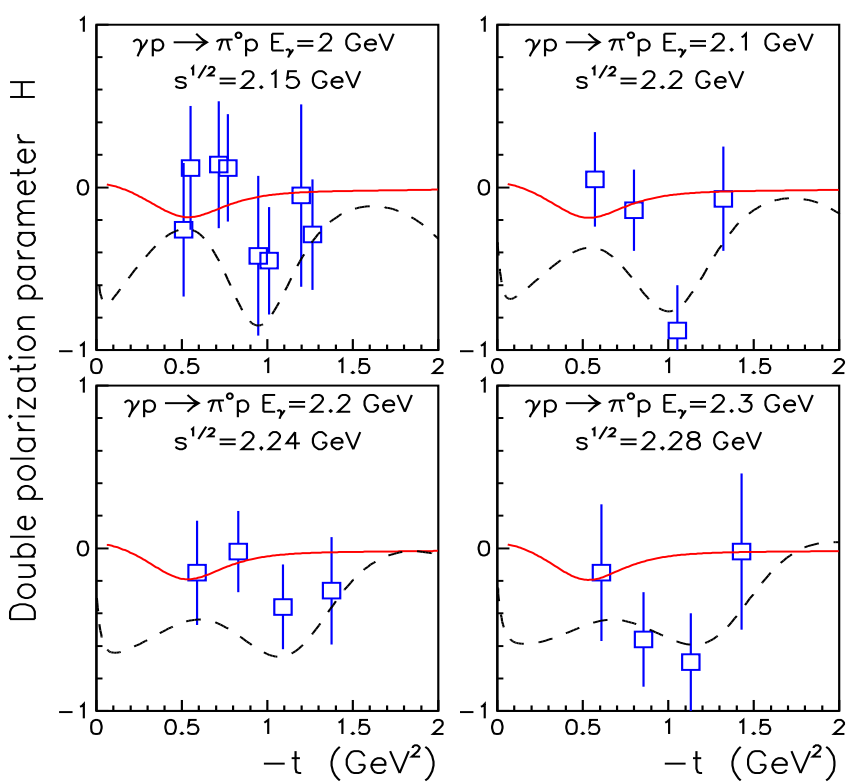

Fig. 9. Double polarization parameter $H$ for $\gamma p \rightarrow \pi^{0} p$ reaction at different photon energies $E_{\gamma}$. The data are from Ref. [74]. The solid lines show the results of our model calculation. The dashed lines are the results based on the GWU PWA [69].

Some comments with regard to the observed difference between $T$ and $P$ at those energies seem to be in order. In the case of $t$-channel non-resonant contributions the $F_{4}$ helicity amplitude is given by higher order corrections and thus we neglect it, as was discussed previously [1]. But even if $F_{4}$ does not vanish its general influence on the various observables is expected [24] 71] to be small. Note that in approaches based on an effective Lagrangian, which are commonly used at low energies, the contribution from vector-meson exchanges to pseudoscalar meson photoproduction also results in a vanishing invariant amplitude $A_{3}$ and, thus, following Eq. (22), one would expect $F_{4}=0$. However, at the same time resonances can contribute [72,73] to the amplitude $F_{4}$ so that the difference between $T$ and $R$ might be explained in a natural way.

For the $\gamma p \rightarrow \pi^{0} p$ reaction there are also data [74] for the double polarization parameters $G$ and $H$. These data are important for fixing the sign of the amplitude $F_{2}$, as is obvious from Eqs. (19) and (20). Since there are no data for the double polarization parameters at higher energies we cannot determine the sign of the $F_{2}$ amplitude within our fitting procedure. Therefore, we decided to use the data at low energies to fix that ambiguity for the parameters corresponding to the $F_{2}$ amplitude, listed in the Table 2 .

Fig. 8 shows the double polarization parameter, $G$, measured at photon energies from 2 to $2.3 \mathrm{GeV}$, which correspond to invariant energies of 2.15 to $2.28 \mathrm{GeV}$. The solid lines are the results obtained from our Regge model. They are only very qualitatively in line with the data. A similar conclusion might also be drawn when comparing the PWA results with the measurements.

Fig. 9 shows the double polarization parameter, $H$, measured for photon energies between 2 and $2.3 \mathrm{GeV}$. The solid lines are the results obtained from the Regge model. Again,

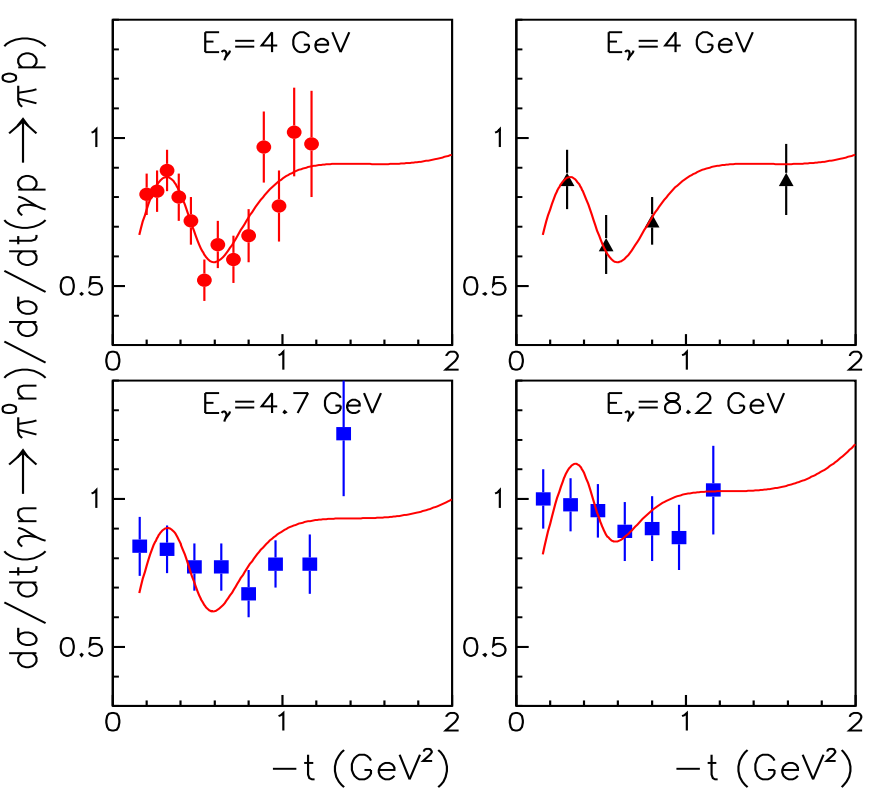

Fig. 10. The ratio of the differential cross sections for $\pi^{0}-$ photoproduction on neutrons and protons as a function of $-t$ for different photon energies $E_{\gamma}$. The data are from Refs. [78] (circles), [79] (triangles) and [80] (squares). The lines show the results of our model calculation.

these are qualitatively in line with the experiment. It is interesting that the PWA predicts large negative values for $H$ that vary strongly with the four-momentum momentum squared, while the Regge model predicts $H \simeq 0$.

\section{Further results and discussion}

\subsection{Total cross sections and the ratio of $\pi^{0}$ photoproduction on neutrons and protons}

Information about the relative contributions of the isovector $\rho$ (and $b_{1}$ ) exchange and the isoscalar $\omega$ exchange amplitudes can be obtained by comparing the differential cross sections for $\pi^{0}$ meson photoproduction on neutrons and on protons [25, 75, 76, 77]. For the proton target the total reaction amplitude is given by the sum of the isovector and isoscalar contributions, while for the neutron target it is given by their difference.

In Fig. 10 we present the ratio of the differential cross sections for $\pi^{0}$-photoproduction on neutrons and protons for different photon energies. The available data demonstrate that the ratio $R \neq 1$, which contradicts a statement given in Ref. [40]. Note that the precise measurement [78] at $E_{\gamma}=4 \mathrm{GeV}$ indicates that the ratio depends considerably on $t$.

Fig. 11] shows the total cross section for the $\gamma p \rightarrow \pi^{0} p$ reaction as a function of the invariant collision energy. The experimental results were obtained [3] by integration over the angular distributions and involve an extrapolation into the forward and backward regions using the results from the isobar model of Anisovich et al. [81]. The solid line is the Regge result, obtained by integration of the calculated differential cross section over the range $|t| \leq 2 \mathrm{GeV}^{2}$. 


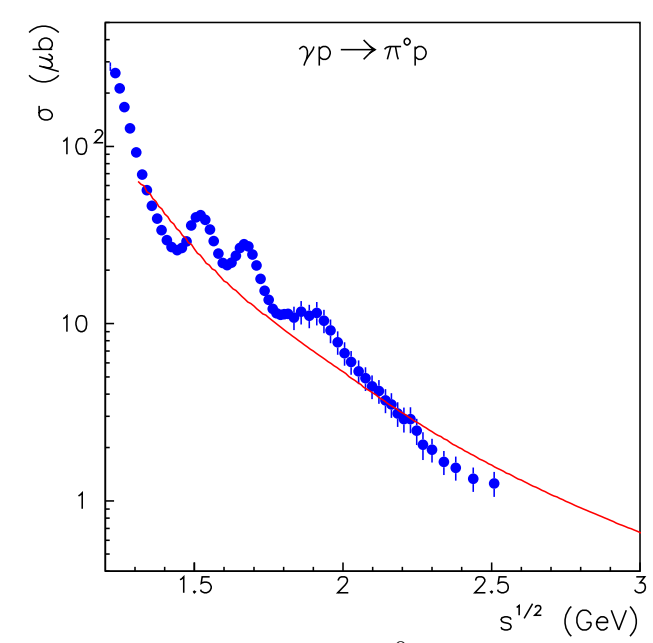

Fig. 11. Total cross section for $\gamma p \rightarrow \pi^{0} p$. The circles are the experimental results taken from Ref. [3]. The lines show the results of our model calculation.
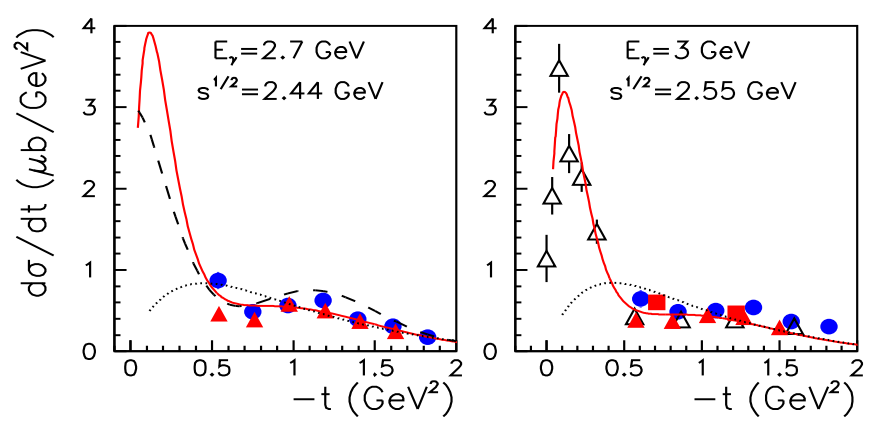

Fig. 12. Differential cross section for $\gamma p \rightarrow \pi^{0} p$ as a function of $-t$ at $E_{\gamma}=2.7$ and $3 \mathrm{GeV}$. The data are taken from Refs. [2] (filled triangles), [3] (filled circles), [54] (open triangles) and [55 56] (filled squares). The solid lines show the results of our model calculation. The dashed line is the result based on the GWU PWA [69] while the dotted line is the result of the isobar model [81] as given in Ref. [3].

At first sight it looks as if the Regge model would overestimate the integrated cross section significantly at the higher energies, i.e. at energies where it is actually expected to agree with the data. However, the amplitude that is used for obtaining those cross sections in [3] has some shortcomings in the forward direction, as one can see in Fig. 12. Specifically, it fails badly to describe the data at very small angles [54], whereas the Regge model reproduces even those data rather well. Consequently, a determination of the total cross section that utilizes that amplitude for extrapolating to forward angles will necessarily underestimate the "real" value. On the contrary, based on the quality of our fit to the small-angle data at $3 \mathrm{GeV}$, one expects that the predictions of the Regge model for the integrated cross section should be very realistic. It is interesting to see that the results of the isobar model [81] and of our Regge fit practically coincide in the range $1<-t<2 \mathrm{GeV}^{2}$.

\subsection{Results for fixed four-momentum transfer}

To complete our analysis of the data we take a look at the energy dependence of the $\gamma p \rightarrow \pi^{0} p$ differential cross sections at fixed four-momentum transfer squared $t$. This allows us to shed light on the applicability of the Regge phenomenology with respect to the $s$ - as well as the $t$ dependence. It also facilitates the inspection as to whether potential discrepancies between the calculations and data exhibit any systematic features.

Fig. 13 shows the data considered in the present analysis. Here the differential cross sections are multiplied by the squared invariant collision energy $s$. We multiply with this factor because the high energy limit of the cross section at small $-t$, as given by the Regge formalism, is proportional to $1 / s$. Therefore, at high energies and small $-t$ we expect that $s d \sigma / d t$ approaches a constant value. For ease of comparison we scale the data and the curves by powers of 10 .

The solid lines in Fig. 13] are the results of our Regge model. At very small $|t|$ the Regge model reproduces the data rather well, even down to energies of $\sqrt{s} \simeq 2 \mathrm{GeV}$. In general the data seem to agree with the high energy limit as given by the Regge phenomenology from energies of $\sqrt{s}=2.5-3 \mathrm{GeV}$ upwards, at least for the range $0.5 \leq|t| \leq 2 \mathrm{GeV}^{2}$. Below this energy region the data show sizeable variations with regard to the predictions of the Regge model. Specifically, for low energies and larger $|t|$ our model results deviate systematically from the data and the discrepancy increases with increasing squared four-momentum transfer.

Furthermore, as the squared four-momentum increases the energy dependence of the data and the Regge calculations becomes steeper. Although we fit the data in the range $t \geq-2$ $\mathrm{GeV}$, the calculations still describe the experimental results at $t=-2.5 \mathrm{GeV}$ fairly well. However, the Regge model substantially underestimates all data at larger $|t|$. As we showed in Ref. [1], the data on charged pion photoproduction at energies $\sqrt{s} \geq 2.7 \mathrm{GeV}$ and at large four-momentum transfer squared are practically independent of $t$ and are in line with the Dimensional Counting Rule [82,83]. According to the DCR for the invariant amplitude $M$ the energy dependence of the differential cross-section is given as

$$
\frac{d \sigma}{d t}=\frac{|M|^{2} F(t)}{16 \pi\left(s-m_{N}^{2}\right)^{2}}=\frac{c s^{-\left(n_{i}-2\right)\left(n_{f}-2\right)} F(t)}{16 \pi s^{2}} \propto c s^{-7} F(t),(11)
$$

in the limit $m_{N} \ll s$, where $m_{N}$ is the mass of the nucleon. Here $c$ is a normalization constant, while $n_{i}$ and $n_{f}$ are the total number of elementary fields in the initial and final states, respectively. For single pion photoproduction $n_{i}=4$ and $n_{f}=5$. Furthermore, $F(t)$ is a form-factor, which does not depend on the energy $s$ but accounts for the $t$ dependence of the hadronic wave functions and partonic scattering. We found that, within the experimental uncertainties, the data on $\pi^{+}$and $\pi^{-}$-meson photoproduction indicate that $F(t)$ is almost constant, i.e. does not depend on the squared four momentum. Moreover, it turns out that both negative and positive pion photoproduction can be described with the same normalization $c=11 \mathrm{mb} \cdot \mathrm{GeV}^{12}$, when assuming that $F(t)=1$.

The dashed lines in Fig. 13 show the results obtained using Eq. (11) with the normalization constant given above. Apart from two experimental points at $\sqrt{s} \geq 5 \mathrm{GeV}$ the data are in 

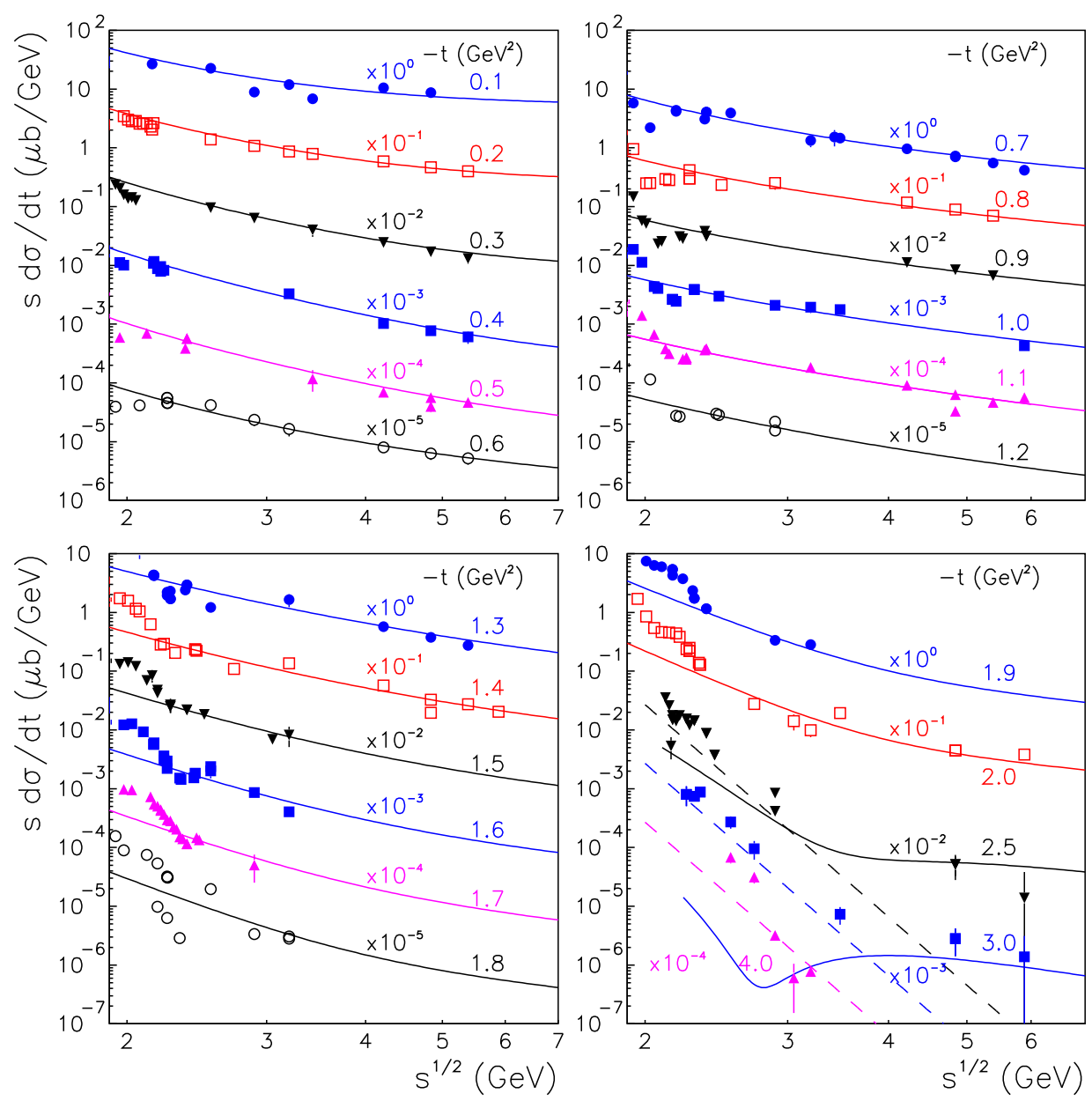

Fig. 13. Differential cross section for $\gamma p \rightarrow \pi^{0} p$ as a function of the invariant collision energy, at selected fixed values of $t$. The symbols show the data considered in the present paper. The solid lines are the results of our model calculation. The dashed lines are the results obtained with Eq. (11). The data and lines are scaled with powers of 10.

good agreement with the ansatz based on the DCR. However, note that at least at $t=-2.5 \mathrm{GeV}^{2}$ the Regge calculation reproduces the data better than the DCR.

\section{Primakoff effect}

The Primakoff effect [5] has not only been observed in neutral pion photoproduction on nuclei but also in the $\gamma p \rightarrow \pi^{0} p$ reaction [54]. This effect dominates the reaction cross section at low momentum transfer and can be used for the determination of the $\pi^{0} \rightarrow \gamma \gamma$ decay width. But there is an interference of the $F_{1}$ amplitude with the Primakoff (one photon exchange) amplitude. Thus, the determination of the $\pi^{0}$ radiative decay requires a precise knowledge of the hadronic part of the amplitude as well as accurate data. Unfortunately, the available experimental results [54] on the differential cross section in the near forward direction are afflicted by considerable uncertainties, as is illustrated in Fig. 14. Here the dotted lines show the result of our Regge model without one photon exchange, which reproduces the data at angles above $10^{\circ}$, say, rather well.
The solid lines in Fig. 14 are results obtained with the Primakoff amplitude included, where the latter is given by

$$
F^{P}=\frac{8 m_{p}}{t} \sqrt{\frac{\pi \Gamma\left(\pi^{0} \rightarrow \gamma \gamma\right)}{m_{\pi}^{3}}} F_{D}(t)=\sqrt{\Gamma} \hat{F}^{P}(t) .
$$

Here $m_{p}$ and $m_{\pi}$ are the proton and pion mass, respectively, $\Gamma$ is the $\pi^{0} \rightarrow \gamma \gamma$ decay width and $F_{D}$ is the Dirac form factor of the proton. For the latter we adopt the parameterization given in Ref. [84],

$$
F_{D}(t)=\frac{4 m_{p}^{2}-2.8 t}{4 m_{p}^{2}-t} \frac{1}{\left(1-t / t_{0}\right)^{2}}
$$

with $t_{0}=0.71 \mathrm{GeV}^{2}$, which is derived under the assumptions that the Dirac form factor $F_{D}$ of the neutron and the isoscalar Pauli form factor vanish and that a dipole form is satisfactory for $G_{M} \approx \mu G_{E}(t)$, cf. [84]. There are slight deviations from the dipole form in the region $-t<0.5 \mathrm{GeV}^{2}$ we are concerned with here, cf. for example Ref. [85], but we neglect those in the present exploratory study. The amplitude of Eq. (12) is added to the helicity amplitude $F_{1}$ of our Regge model. We show results 

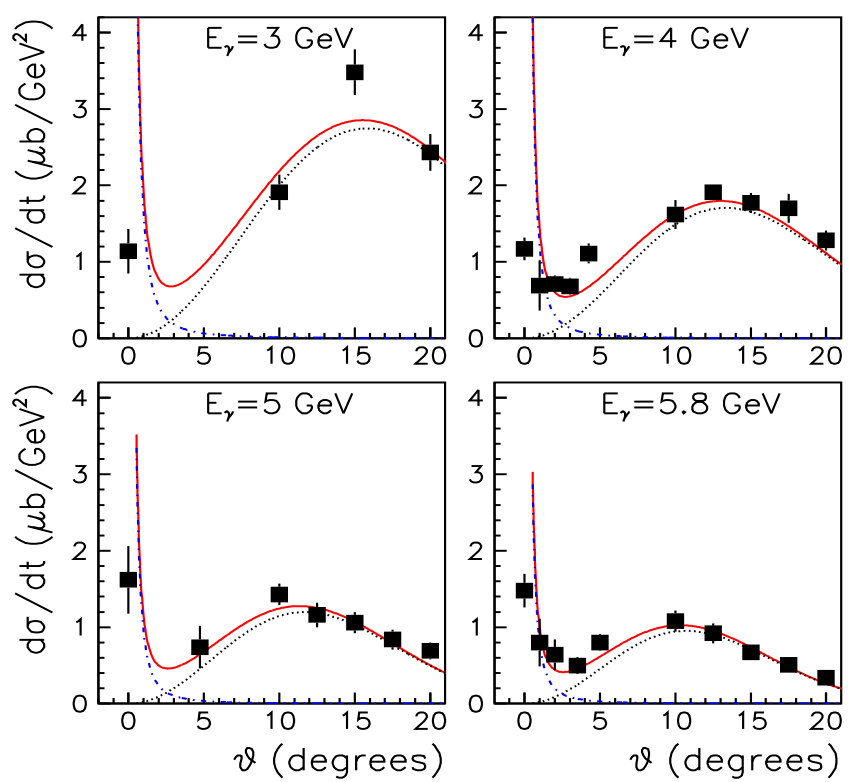

Fig. 14. Differential cross section for $\gamma p \rightarrow \pi^{0} p$ as a function of the angle $\theta$ in the $\mathrm{cm}$ system shown for different photon energies $E_{\gamma}$. The data are taken from Ref. [54]. The dotted lines show the Regge calculations without one photon exchange, while the solid lines are the results obtained with inclusion of the one photon exchange. The dash-dotted lines are results for the one photon exchange alone.

based on $\Gamma\left(\pi^{0} \rightarrow \gamma \gamma\right)=8.4 \mathrm{eV}$, i.e. the value that is given by the PDG as the average $\pi^{0}$-meson lifetime. The calculations are not folded with the pertinent angular resolution function [54], because this quantity is is not available to us for the particular experiment in question.

Fig. 14illustrates impressively the consequences of the Primakoff effect. As demonstrated in the preceeding sections, the $\gamma p \rightarrow \pi^{0} p$ reaction amplitude can be well fixed by the huge set of data available at larger angles $\theta>10^{\circ}$ and at different photon energies. This ensures that the hadronic contribution to the photoproduction amplitude is known quite precisely when extrapolating to forward angles. Apparently, the situation is different for measurements on nuclear targets. In that case the reactions at large angles are entirely dominated by incoherent photoproduction and it is very difficult to fix the coherent nuclear amplitude, which contributes at forward angles [86, 87, 88, 89, 90]. Thus, $\pi^{0}$-meson photoproduction on the proton could offer a promising alternative for the determination of the $\pi^{0}$ radiative decay width. We should emphasize, however, that for the reaction on the proton the relative phase between the hadronic part and the Primakoff amplitude is also an unknown quantity. In the present exploratory calculation we have simply added the latter as given in Eq. (12) to our Regge amplitude. But in a concrete application to experimental data one needs to determine this phase together with the $\pi^{0}$ radiative decay width by a fit to differential cross sections at forward angles.

For completeness we also show the $\gamma p \rightarrow \pi^{0} p$ differential cross section at forward angles for the photon energy $E_{\gamma}=2 \mathrm{GeV}$, cf. Fig. 15. Here the squares are data from Ref. [54], triangles are the results from the CLAS experiment [2] and circles are data from the CB-ELSA Collaboration [3]. Unfortunately, the

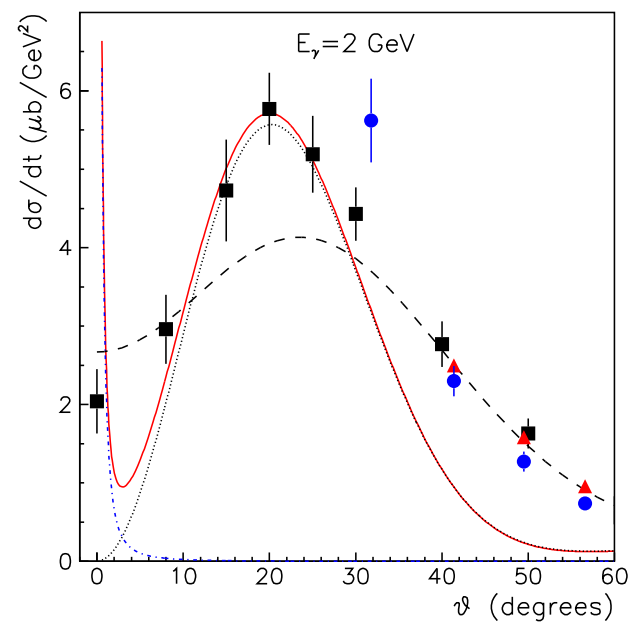

Fig. 15. Differential cross section for $\gamma p \rightarrow \pi^{0} p$ as a function of the angle $\theta$ in the $\mathrm{cm}$ system shown for photon energy $E_{\gamma}=2 \mathrm{GeV}$. The data are taken from Refs. [54] (squares), [2] (triangles) and [3] (circles). The dotted line shows the Regge calculations without one photon exchange, while the solid line is the results obtained with inclusion of one photon exchange. The dash-dotted line is the result for one photon exchange alone. The dashed line indicates the results based on the GWU PWA [69].

latter recent measurements [2,3] do not cover the region of very forward angles. The dotted line in Fig. 15] is the result of the Regge model alone while the solid line was obtained with inclusion of the Primakoff amplitude. Our model reproduces the data at forward angles surprisingly well, but it underestimates the experimental results at $\theta>30^{\circ}$. The dashed line indicates results based on the current solution of the GWU PWA [68],
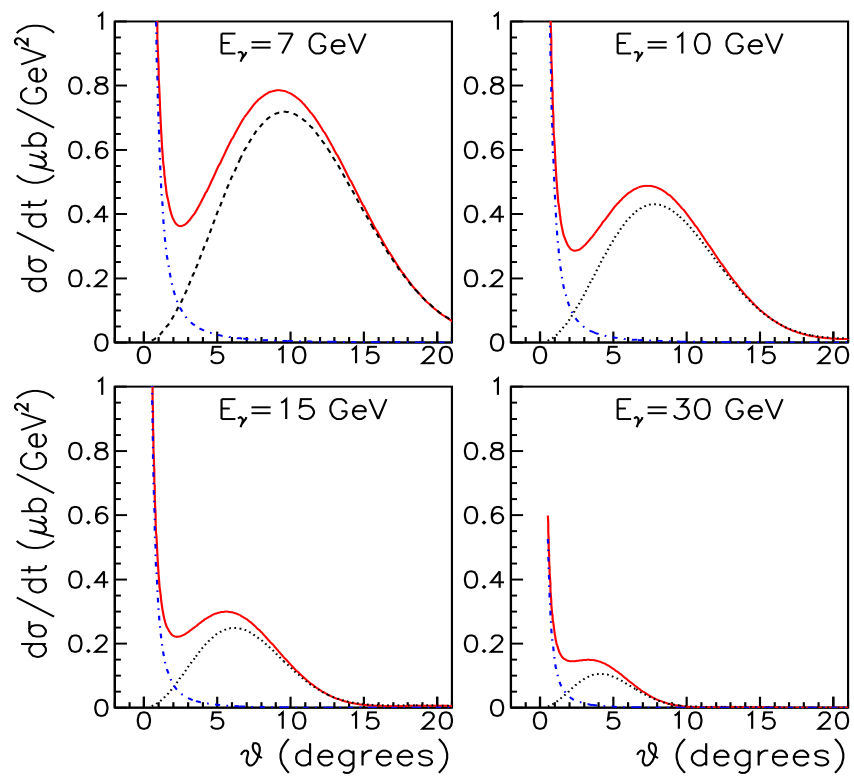

Fig. 16. Differential cross section for $\gamma p \rightarrow \pi^{0} p$ as a function of the angle $\theta$ in the $\mathrm{cm}$ system shown for different photon energies $E_{\gamma}$. Same description of curves as in Fig. 14 


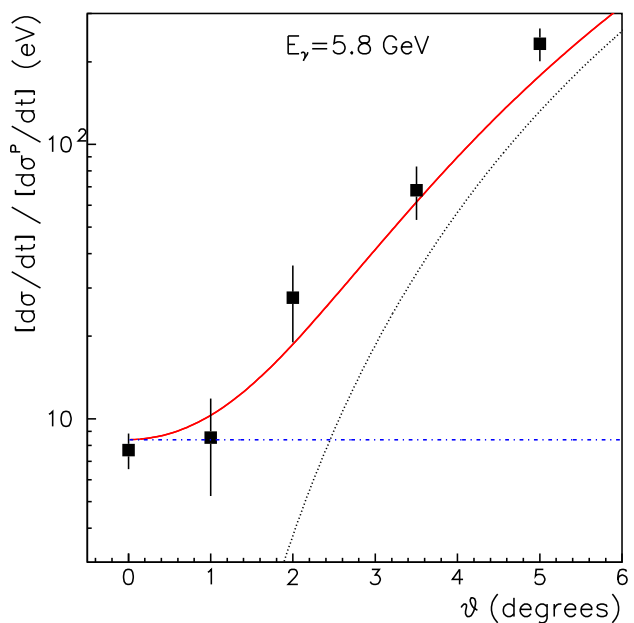

Fig. 17. Differential cross section for $\gamma p \rightarrow \pi^{0} p$ at $E_{\gamma}=5.8 \mathrm{GeV}$ divided by $d \sigma^{P} / d t$ (Eq. (14)). The data are from Refs. [54]. Same description of curves as in Fig. 14

which describes the data at $\theta \geq 40^{\circ}$ but does not reproduce the $\gamma p \rightarrow \pi^{0} p$ differential cross section at forward angles.

Fig.[15illustrates an interesting feature. The excellent agreement of our Regge calculation with the available data at forward angles could be an indication that even at such low energies the forward photoproduction is still dominated by $t$-channel contributions. If so then there would be indeed very good conditions for determining the $\pi^{0}$-meson lifetime from measurements of neutral pion photoproduction at photon energies around $E_{\gamma} \simeq 2 \mathrm{GeV}$ that are accessible presently at Jlab and ELSA.

Predictions for higher energies are presented in Fig. 16 Experiments in this energy region will become feasibly once JLab's $12 \mathrm{GeV}$ Upgrade Project will be completed.

Finally, let us provide another view on the present situation. In Fig. 17] we show again the available cross-section data at $5.8 \mathrm{GeV}$ [54], but divide the data and the curves by the contribution of the pure Primakoff amplitude with a normalization so that the result at zero angle coincides with the $\pi^{0}$ decay width, i.e. we divide by

$$
\frac{d \sigma^{P}}{d t}=\frac{1}{32 \pi}\left[\frac{t\left|\hat{F}^{P}\right|^{2}}{\left(t-4 m_{p}^{2}\right)}\right] .
$$

Note that a logarithmic scale is used for the ordinate. On this plot one can see down to which angles the results are still dominated by the hadronic amplitude. It is obvious that an appropriate set of data points below 3 degrees, say, and with high precision would allow an extrapolation to zero degrees and, thus, a determination of $\Gamma\left(\pi^{0} \rightarrow \gamma \gamma\right)$. The presently available data are too sparse and too inaccurate for performing such an extrapolation reliably.

\section{Summary}

In the present paper we performed a global analysis of the world data on the reactions $\gamma p \rightarrow \pi^{0} p$ and $\gamma n \rightarrow \pi^{0} n$ for photon energies from 3 to $18 \mathrm{GeV}$ within the Regge approach. In this region resonance contributions are expected to be negligible so that the available experimental information on differential cross sections and single- and double polarization observables at $-t \leq 2 \mathrm{GeV}^{2}$ allows us to determine the reaction amplitude reliably. The Regge model was constructed by taking into account both pole and cut exchange $t$-channel helicity amplitudes and includes the $\rho, \omega$ and $b_{1}$ trajectories. The model parameters such as the helicity couplings were fixed by a fit to the available data in the considered $E_{\gamma}$ and $t$ range.

An excellent overall description of the available data was achieved, indicating that for the energy and $t$ range in question single pion photoproduction is indeed dominated by nonresonant contributions. The model amplitude was then used to predict observables for photon energies below $3 \mathrm{GeV}$. A detailed comparison with recent data from the CLAS (JLab) and CBELSA (Bonn) Collaborations in that energy region was presented. It turned out that the resulting differential cross sections for $\gamma p \rightarrow \pi^{0} p$ were still in reasonable agreement with those new data down to $E_{\gamma} \approx 2.45 \mathrm{GeV}$ for $-t \leq 2 \mathrm{GeV}^{2}$, while the very forward data were reproduced even down to photon energies as low as $2 \mathrm{GeV}$.

Since our Regge amplitude works so well for forward angles, even at very low energies, we utilized it to explore the prospects for determining the $\pi^{0}$ radiative decay width via the Primakoff effect from the reaction $\gamma p \rightarrow \pi^{0} p$. Those calculations indicate that corresponding measurements on a proton target could be indeed promising. But, evidently, the precision to which the decay width can be determined will depend crucially on the number of data points that one can collect at very small angles and on the accuracy and the angular resolution one can achieve.

\section{Acknowledgements}

We acknowledge fruitful discussions with A. Bernstein, W. Chen, M. Dugger, L. Gan, H. Gao, A. Gasparian, J. Goity, I. Strakovsky, and U. Thoma. This work is partially supported by the Helmholtz Association through funds provided to the virtual institute "Spin and strong QCD" (VH-VI-231), by the European CommunityResearch Infrastructure Integrating Activity "Study of Strongly Interacting Matter" (acronym HadronPhysics2, Grant Agreement no. 227431) under the Seventh Framework Programme of EU, and by DFG (SFB/TR 16, "Subnuclear Structure of Matter"). This work was also supported in part by U.S. DOE Contract No. DE-AC05-06OR23177, under which Jefferson Science Associates, LLC, operates Jefferson Lab. A.S. acknowledges support by the JLab grant SURA-06-C0452 and the COSY FFE grant No. 41760632 (COSY-085). 


\section{Appendix A}

Utilizing the relations of Ref. [91] the $\gamma N \rightarrow \pi N$ observables analysed in our study are given in terms of the amplitudes $F_{i}$ $(i=1, \ldots, 4)$ by [38]

$$
\begin{aligned}
\frac{d \sigma}{d t} & =\frac{1}{32 \pi}\left[\frac{t\left|F_{1}\right|^{2}-\left|F_{3}\right|^{2}}{\left(t-4 m_{N}^{2}\right)}+\left|F_{2}\right|^{2}-t\left|F_{4}\right|^{2}\right] \\
\frac{d \sigma}{d t} \Sigma & =\frac{1}{32 \pi}\left[\frac{t\left|F_{1}\right|^{2}-\left|F_{3}\right|^{2}}{\left(t-4 m_{N}^{2}\right)}-\left|F_{2}\right|^{2}+t\left|F_{4}\right|^{2}\right] \\
\frac{d \sigma}{d t} T & =\frac{\sqrt{-t}}{16 \pi} \operatorname{Im}\left[\frac{-F_{1} F_{3}^{*}}{\left(t-4 m_{N}^{2}\right)}+F_{4} F_{2}^{*}\right] \\
\frac{d \sigma}{d t} P & =\frac{\sqrt{-t}}{16 \pi} \operatorname{Im}\left[\frac{-F_{1} F_{3}^{*}}{\left(t-4 m_{N}^{2}\right)}-F_{4} F_{2}^{*}\right] \\
\frac{d \sigma}{d t} G & =\frac{\operatorname{Im}\left[t F_{4}\left(F_{3}^{*}-2 m_{N} F_{1}^{*}\right)+F_{2}\left(t F_{1}^{*}-2 m_{N} F_{3}^{*}\right)\right]}{16 \pi\left(t-4 m_{N}^{2}\right)} \\
\frac{d \sigma}{d t} H & =\frac{\sqrt{-t} \operatorname{Im}\left[F_{2}\left(F_{3}^{*}-2 m_{N} F_{1}^{*}\right)+F_{4}\left(t F_{1}^{*}-2 m_{N} F_{3}^{*}\right)\right]}{16 \pi\left(t-4 m_{N}^{2}\right)}
\end{aligned}
$$

In order to account for the correct behavior at very small angles $t$ should be replaced by $t-t_{\min }$ in the above formulae, where $t_{\text {min }}=-\left(m_{\pi^{0}} / 2 E_{\gamma}\right)^{2}$.

\section{Appendix B}

Here we provide the relation between the $t$-channel helicity amplitudes $F_{i}$ and the $s$-channel helicity amplitudes $S_{1}, S_{2}$, $N$ and $D$. Following Wiik's abbreviations [92], $S_{1}$ and $S_{2}$ are single spin-flip amplitudes, $N$ is the spin non-flip and $D$ is the double spin-flip amplitude, respectively. The asymptotic crossing relation, which is useful for the analytical evaluation of the helicity amplitudes, is given by

$$
\left[\begin{array}{c}
F_{1} \\
F_{2} \\
F_{3} \\
F_{4}
\end{array}\right]=\frac{-4 \sqrt{\pi}}{\sqrt{-t}}\left[\begin{array}{cccc}
2 m_{N} & \sqrt{-t} & -\sqrt{-t} & 2 m_{N} \\
0 & \sqrt{-t} & \sqrt{-t} & 0 \\
t & 2 m_{N} \sqrt{-t} & -2 m_{N} \sqrt{-t} & t \\
1 & 0 & 0 & -1
\end{array}\right]\left[\begin{array}{c}
S_{1} \\
N \\
D \\
S_{2}
\end{array}\right] .
$$

Note that Eq. (21) is appropriate only at $s \gg t$, since it does not account for higher order corrections that are proportional to $t / 4 m_{N}^{2}$. The amplitudes $F_{i}$ are related to the usual CGLN invariant amplitudes $A_{i}[93]$ by

$$
\begin{aligned}
& F_{1}=-A_{1}+2 m_{N} A_{4}, \\
& F_{2}=A_{1}+t A_{2}, \\
& F_{3}=2 m_{N} A_{1}-t A_{4}, \\
& F_{4}=A_{3} .
\end{aligned}
$$

Expressions for the experimental observables in terms of the amplitudes $A_{i}$ are listed, for instance, in Ref. [94]. The often used multipole amplitudes can be constructed from the helicity amplitudes using the relations given in Refs. [95, 96, 97].

\section{References}

1. A. Sibirtsev, J. Haidenbauer, S. Krewald, T.S.H. Lee, Ulf-G. Meißner and A.W. Thomas, Eur. Phys. J. A 34, 49 (2007) arXiv:0706.0183].

2. M. Dugger et al., Phys. Rev. C 76, 025211 (2007) [arXiv:0705.0816].

3. O. Bartholomy et al., Phys. Rev. Lett. 94, 012003 (2005); [hep-ex/0407022].

4. H. van Pee et al., Eur. Phys. J. A 31, 61 (2007) |arXiv:0704.1776|.

5. H. Primakoff, Phys. Rev. 81, 899 (1951).

6. S. L. Adler, Phys. Rev. 177, 2426 (1969).

7. J. S. Bell and R. Jackiw, Nuovo Cim. A 60, 47 (1969).

8. W. A. Bardeen, Phys. Rev. 184, 1848 (1969).

9. J. Wess and B. Zumino, Phys. Lett. B 37, 95 (1971).

10. E. Witten, Nucl. Phys. B 223, 422 (1983).

11. J. Bijnens, Int. J. Mod. Phys. A 8, 3045 (1993).

12. J. L. Goity, A. M. Bernstein and B. R. Holstein, Phys. Rev. D 66, 076014 (2002) [arXiv:hep-ph/0206007].

13. K. Kampf and B. Moussallam, arXiv:0901.4688 [hep-ph].

14. C.A. Engelbrecht, Phys. Rev. 133, B988 (1964)

15. G. Bellettini et al., Nuovo Cim. A 66, 243 (1970).

16. V.I. Kryshkin, A.G. Sterligov, and Yu.P. Usov, Izv. Vuz. Fiz. 6 , 101 (1970).

17. V.I. Kryshkin, A.G. Sterligov, and Yu.P. Usov, JETP 30, 1037 (1970)

18. A. Browman et al., Phys. Rev. Lett. 33, 1400 (1974).

19. E. Hadjimichael, Phys. Rev. C 39, 1438 (1989).

20. C. Amsler et al., Phys. Lett. B 667 , 1 (2008).

21. A. Gasparian et al., Proposal E-02-103 (2001).

22. T.E. Rodrigues, J.D.T. Arruda-Neto, J. Mesa, C. Garcia, K. Shtejer, D. Dale and I. Nakagawa, Phys. Rev. C 71, 051603 (2005).

23. J. M. Laget, Phys. Rev. C 72, 022202(R) (2005).

24. A. C. Irving and R. P. Worden, Phys. Rept. 34, 117 (1977).

25. A. C. Irving and L.G.F. Vanryckeghem, Nucl. Phys. B 93, 324 (1975).

26. N.J. Sopkovitch, Nuovo Cim. 26, 186 (1962).

27. K. Gottfried and J.D. Jackson, Nuovo Cim. 34, 735 (1964).

28. J.D. Jackson, Rev. Mod. Phys. 42, 12 (1970).

29. R. Worden, Nucl. Phys. B 37, 253 (1972).

30. R.C. Arnold, Phys. Rev. 153, 1523 (1967).

31. J. D. Jackson and C. Quigg, Phys. Lett. B 29, 236 (1969).

32. V.M. Gribov, I.Y. Pomeranchuk and K.A. Ter-Martirosyan, Phys. Rev. 139B, 184 (1965).

33. A.R. White, Nucl. Phys. B 50, 93 (1972).

34. A.R. White, Nucl. Phys. B 50, 130 (1972).

35. P.D.B. Collins, An Introduction to Regge Theory and High Energy Physics, Cambridge University, Cambridge, England (1977) 275.

36. P.D.B. Collins and A.D. Martin, Rept. Prog. Phys. 45, 335 (1982).

37. F. Huang, A. Sibirtsev, S. Krewald, C. Hanhart, J. Haidenbauer, Ulf-G. Meißner, arXiv:0810.2680, Eur. Phys. J. A, in print.

38. I. S Barker, A. Donnachie and J. K. Storrow, Nucl. Phys. B 79, 431 (1974).

39. B. H. Kellett, Nucl. Phys. B 25, 205 (1970).

40. M. Guidal, J.M. Laget and M. Vanderhaeghen, Nucl. Phys. A 627, 645 (1997).

41. M. Vanderhaeghen, M. Guidal and J.M. Laget, Phys. Rev. C 57, 1454 (1998).

42. K. Nakayama, and W.G. Love, Phys. Rev. C 70, 012201 (2004) [arXiv:hep-ph/0404011].

43. D. Bellenger et al., Phys. Rev. Lett. 23, 540 (1969).

44. R. L. Anderson et al., Phys. Rev. D 4, 1937 (1971).

45. P.S.L. Booth et al., Phys. Lett. B 38, 339 (1972). 
46. H. Bienlein et al., Phys. Lett. B 46, 131 (1973).

47. M. Deutsch et al., Phys. Rev. Lett. 29,1752 (1972).

48. A. Sibirtsev, K. Tsushima and S. Krewald, Phys. Rev. C 67, 055201 (2003) [arXiv:nucl-th/0301015].

49. M. Rahnama and J.K. Storrow, J. Phys. G 17, 243 (1991).

50. J.N.J. White, Phys. Lett. B 26, 461 (1968).

51. J.N.J. White, Nucl. Phys. B 13, 139 (1969).

52. F. Henyey, G. L. Kane, Jon Pumplin and M. H. Ross, Phys. Rev. 182, 1579 (1969).

53. G.C. Bolon et al., Phys. Rev. Lett. 18, 926 (1967).

54. M. Braunschweig et al., Nucl. Phys. B 20, 191 (1970).

55. M.A. Shupe et al., Phys. Rev. Lett. 40, 271 (1978).

56. M.A. Shupe et al., Phys. Rev. D 19, 1921 (1979).

57. R. L. Anderson et al., Phys. Rev. D 1, 27 (1970).

58. B. Barish et al., Phys. Rev. D 9, 566 (1974).

59. The Durham High Energy Physics Databases; the data are available on the web: http: //durpdg.dur.ac.uk/HEPDATA/.

60. G. Höhler, $\pi N$ Newsletter 9, 1 (1993).

61. G. Höhler, Landolt-Börnstein 9, Springer, Berlin, 1983.

62. R. Koch, Nucl. Phys. A 448, 707 (1986).

63. R. E. Cutkovsky et. al., Phys. Rev. D 20, 2839 (1980).

64. D. M. Manley and E. M. Saleski, Phys. Rev. D 45, 4002 (1992).

65. R. A. Arndt, R. L. Workman, Z. Li and L. D. Roper, Phys. Rev. C 42, 1853 (1990) .

66. R. A. Arndt et. al., Phys. Rev. D 43, 2131 (1991).

67. R. Arndt, W.J. Briscoe, I.I. Strakovsky and R.L. Workman, Phys. Rev. C 66, 055213 (2002).

68. R. Arndt, W.J. Briscoe, I.I. Strakovsky and R.L. Workman, Eur. Phys. J. A 35, 311 (2008).

69. R.A. Arndt, W.J. Briscoe, R.L. Workman and I.I. Strakovsky, CNS Data Analysis Center, http://gwdac.phys.gwu.edu/

70. P.J. Bussey et al., Nucl. Phys. B 104, 253 (1976).

71. M. Rahnama and J. K. Storrow, Z. Phys. C 10, 263 (1981).

72. M. Benmerrouche and N.C. Mukhopadhyay, Phys. Rev. D 51, 3237 (1995) [arXiv:hep-ph/9412248].

73. A. Sibirtsev, C. Elster, S. Krewald and J. Speth, AIP Conf. Proc. 717, 837 (2004) |arXiv:nucl-th/0303044|.

74. P.J. Bussey et al., Nucl. Phys. B 159, 383 (1979).

75. I.S. Barker and J.K. Storrow, Nucl. Phys. B 137, 413 (1978).

76. J. Froyland, Nucl. Phys. B 11, 204 (1969).

77. E.N. Argyres, A.P. Contogouris, S. Ray and M.Svec, Nucl. Phys. B 45, 267 (1972).

78. W. Braunschweig et al., Nucl. Phys. B 51, 157 (1973).

79. G.C. Bolon et al., Phys. Rev. Lett. 27, 964 (1971).

80. A.M. Osborne et al., Phys. Rev. Lett. 29, 1621 (1972).

81. A. Anisovich, E. Klempt, A. Sarantsev and U. Thoma, Eur. Phys. J. A 24, 111 (2005) [arXiv:hep-ph/0407211].

82. S.J. Brodsky and J.R. Farrar, Phys. Rev. Lett. 31, 1153 (1973).

83. V.A. Matveev, R.M. Muradian and A.N. Tavkhelidze, Lett. Nuovo Cimento 7, 7171 (1973).

84. A. Donnachie and P.V. Landshoff, Nucl. Phys. B 231, 189 (1984).

85. M.A. Belushkin, H.-W. Hammer, and Ulf-G. Meißner, Phys. Rev. C 75, 035202 (2007).

86. T.H. Bauer, R.D. Spital and D.R. Yennie, Rev. Mod. Phys. 50, 261 (1978).

87. J.S. Trefil, Phys. Rev. 180, 1379 (1969).

88. J.S. Trefil, Nucl. Phys. B 11, 330 (1969).

89. A. Sibirtsev, H.-W. Hammer, Ulf-G. Meißner and A.W. Thomas, Eur. Phys. J. A 29, 209 (2006) |arXiv:nucl-th/0606044|.

90. A. Sibirtsev, C. Elster and J. Speth, arXiv:nucl-th/0203044.

91. I.S. Barker, A. Donnachie and J.K. Storrow, Nucl. Phys., B 95, 374 (1975).
92. B.H. Wiik, Proc. Int. Symp. on Electron and Photon Interactions at High Energies, Ithaca, New York, Aug 1971, 163.

93. G. F. Chew, M. L. Goldberger, F. E. Low and Y. Nambu, Phys. Rev. 106, 1345 (1957).

94. F. A. Berends, A. Donnachie and D. L. Weaver, Nucl. Phys. B 4, 1 (1967).

95. J.S. Ball, Phys. Rev. 124, 2014 (1961)

96. G. Kramer and P. Stichel, Z. Phys. 178, 519 (1964).

97. R. A. Arndt, R. L. Workman, Z. Li and L. D. Roper, Phys. Rev. C 42, 1853 (1990). 